\title{
Discrete Analytic Functions: An Exposition
}

\author{
László Lovász
}

\begin{abstract}
Harmonic and analytic functions have natural discrete analogues. Harmonic functions can be defined on every graph, while analytic functions (or, more precisely, holomorphic forms) can be defined on graphs embedded in orientable surfaces. Many important properties of the "true" harmonic and analytic functions can be carried over to the discrete setting.
\end{abstract}

\section{Contents}

1. Introduction 241

2. Notation 242

3. Discrete harmonic functions 243

4. Analytic functions on the grid 246

5. Holomorphic forms on maps 250

6. Topological properties 256

7. Geometric connections 259

8. Operations 262

9. An application in computer science:
Global information from local observation

$\begin{array}{lr}\text { Acknowledgement } & 267\end{array}$

$\begin{array}{lr}\text { Appendix } & 267\end{array}$

$\begin{array}{ll}\text { References } & 273\end{array}$

\section{Introduction}

Discrete and continuous mathematics study very different structures, by very different methods. But they have a lot in common if we consider which phenomena they study: Symmetry, dispersion, expansion, and other general phenomena have interesting formulations both in the discrete and continuous setting, and the influence of ideas from one to the other can be most fruitful. One such notion we should more explicitly mention here are discrete harmonic functions, which can be defined on every graph, and have been studied quite extensively. See [23] for a lot of information on harmonic functions on (infinite) graphs and their connections with electrical networks and random walks. In this paper we show that analycity (most 
notably the uniqueness of analytic continuation and the long-range dependence it implies) is an important phenomenon in discrete mathematics as well.

Discrete analytic functions were introduced for the case of the square grid in the 40's by Ferrand [11] and studied quite extensively in the 50's by Duffin [8]. For the case of a general map, the notion of discrete analytic functions is implicit in a paper of Brooks, Smith, Stone and Tutte [5] (cf. section 7.2) and more recent work by Benjamini and Schramm [4]. They were formally introduced recently by Mercat $[\mathbf{1 8}]$.

Discrete analytic functions and holomorphic forms can be defined on orientable maps, i.e., graphs embedded in orientable surfaces. (Much of this could be extended to non-orientable surfaces, but we don't go into this in this paper.) In graphtheoretic terms, they can be defined as rotation-free circulations (which is the same as requiring that the circulation is also a circulation on the dual graph).

Many important properties of the "true" harmonic and analytic functions can be carried over to the discrete setting: maximum principles, Cauchy integrals etc. Some of these translations are straightforward, sometimes it is not so easy to find the right formulation. But discreteness brings in several new aspects as well, like connections with network flows, matroid theory, various embeddings of graphs, tiling the plane by squares, circle representations etc.

Other aspects of analytic functions are worse off. Integration can be defined on the grid [8], but we run into trouble if we want to extend it to more general maps. Mercat [18] introduced a (rather restrictive) condition called "criticality", under which integrals can be defined. Multiplication is problematic even on the grid. Analogues of polynomials and exponential functions can be defined on the grid [8], and can be extended to to critical maps $[\mathbf{1 9}, \mathbf{2 0}]$.

In this paper we start with briefly surveying two related topics: harmonic functions on graphs and discrete analytic functions on grids. This is not our main topic, and we concentrate on some aspects only that we need later. In particular, we show the connection of harmonic functions with random walks, electrical networks and rubber band structures.

We discuss in detail zero-sets of discrete analytic functions, in particular how to extend to discrete analytic functions the fact that a nonzero analytic function can vanish only on a very small connected piece $[\mathbf{2}, \mathbf{3}]$. As an application, we describe a simple local random process on maps, which has the property that observing it in a small neighborhood of a node through a polynomial time, we can infer the genus of the surface.

\section{Notation}

We recall some terminology from graph theory. Let $G=(V, E)$ be a graph, where $V$ is the set of its nodes and $E$ is the set of its edges. An edge of $G$ is a loop, if both endpoints are the same. Two edges are called parallel, if they connect the same pair of nodes. A graph $G$ is called simple, if it has no loops or parallel edges. The set of nodes connected to a given node $v \in V$ (called its neighbors) is denoted by $N(v)$.

A graph is $k$-connected, if deleting fewer than $k$ nodes always leaves a connected graph. 
A directed graph is a graph in which every edges has an orientation. So each edge $e \in E$ has a tail $t_{e} \in V$ and a head $h_{e} \in V$. Our main concern will be undirected graphs, but we will need to orient the edges for reference purposes.

Let $G$ be a directed graph. For each node $v$, let $\delta v \in \mathbb{R}^{E}$ denote the coboundary of $v$ :

$$
(\delta v)_{e}= \begin{cases}1 & \text { if } t_{e}=v \\ -1 & \text { if } h_{e}=v \\ 0 & \text { otherwise }\end{cases}
$$

Thus $|\delta v|^{2}=d_{v}$ is the degree of $v$. We say that a node $v \in V$ is a source [sink] if all edges incident with it are directed away from [toward] the node. Every function $\pi \in \mathbb{R}^{V}$ gives rise to a vector $\delta \pi \in \mathbb{R}^{E}$, where

$$
(\delta \pi)(u v)=\pi(v)-\pi(u) .
$$

In other words,

$$
\delta \pi=\sum_{v} \pi(v) \delta v
$$

For an edge $e$, let $\partial e \in \mathbb{R}^{V}$ be the boundary of $e$ :

$$
(\partial e)_{i}= \begin{cases}1 & \text { in } i=h(e) \\ -1 & \text { in } i=t(e) \\ 0 & \text { otherwise }\end{cases}
$$

For $\phi: E \rightarrow \mathbb{R}$, we define

$$
\partial \phi(v)=(\delta v)^{T} \phi=\sum_{e: t(e)=v} \phi(e)-\sum_{e: h(e)=v} \phi(e)
$$

In other words,

$$
\partial \phi=\sum_{e} \phi(e) \partial e .
$$

We say that $\phi$ satisfies the flow condition at $v$ if $\partial \phi(v)=0$. We say that $\phi$ is a circulation if it satisfies the flow condition at every node $v$. Note that this depends on the orientation of the edges, but if we reverse an edge, we can compensate for it by switching the sign of $\phi(e)$.

\section{Discrete harmonic functions}

3.1. Definition. Let $G=(V, E)$ be a connected graph. A function $f: V \rightarrow \mathbf{C}$ is called harmonic at node $i$ if

$$
\frac{1}{d_{i}} \sum_{j \in N(i)} f(j)=f(i),
$$

and is said to have a pole at $i$ otherwise. Note that the condition can be re-written as

$$
\sum_{j \in N(i)}(f(j)-f(i))=0
$$


More generally, if we also have a "length" $\ell_{i j}>0$ assigned to each edge $i j$, then we say that $f$ is harmonic on the weighted graph $G=(V, E, \ell)$ at node $i$ if

$$
\sum_{j \in N(i)} \frac{f(j)-f(i)}{\ell_{i j}}=0 .
$$

If $S$ is the set of poles of a function $f$, we call $f$ a harmonic function with poles $S$.

In the definition we allowed complex values, but since the condition applies separately to the real and imaginary parts of $f$, it is usually enough to consider real valued harmonic functions.

Proposition 3.1. Every non-constant function has at least two poles.

This follows simply by looking at the minimum and maximum of the function. In fact, the maximum of a function cannot be attained at a node where it is harmonic, unless the same value is attained at all of its neighbors. This argument can be though of as a (very simple) discrete version of the Maximum Principle.

For any two nodes $a, b \in V$ there is a harmonic function with exactly these poles. More generally, we have the following fact.

Proposition 3.2. For every set $S \subseteq V, S \neq \emptyset$, every function $f_{0}: S \rightarrow \mathbf{C}$ has a unique extension to a function $f: V \rightarrow \mathbf{C}$ that is harmonic at each node in $V \backslash S$.

The proof of uniqueness is easy (consider the maximum or minimum of the difference of any two extensions). The existence of the extension follows from any of several constructions, some of which will be given in the next section. Note that the case $|S|=1$ does not contradict Proposition 3.1: the unique extension is a constant function.

If $S=\{a, b\}$, then a harmonic function with poles $S$ is uniquely determined up to scaling by a real number and translating by a constant. There are various natural ways to normalize; we'll somewhat arbitrarily decide on the following one:

$$
\sum_{u \in N(v)}(f(u)-f(v))= \begin{cases}1 & \text { if } v=b \\ -1 & \text { if } v=a \\ 0 & \text { otherwise }\end{cases}
$$

and

$$
\sum_{u} f(u)=0
$$

We denote this function by $\pi_{a b}$. If $e=a b$ is an edge, we also denote this function by $\pi_{e}$.

Expression (4) is equivalent to saying that the function $\delta \pi$ satisfies the flow condition at node $i$ if and only if $\pi$ is harmonic at $i$. Not every flow can be obtained from a harmonic function: for example, a non-zero circulation (a flow without sources and sinks) would correspond to a non-constant harmonic function with no poles, which cannot exist. In fact, the flow obtained by (1) satisfies, for every cycle $C$, the following condition:

$$
\sum_{e \in C} f_{\pi}(e)=0,
$$


where the edges of $C$ are oriented in a fixed direction around the cycle. We could say that the flow is "rotation-free", but we'll reserve this phrase for a slightly weaker notion in section 5 .

3.2. Random walks, electrical networks, and rubber bands. Harmonic functions play an important role in the study of random walks: after all, the averaging in the definition can be interpreted as expectation after one move. They also come up in the theory of electrical networks, and in statics. This provides a connection between these fields, which can be exploited. In particular, various methods and results from the theory of electricity and statics, often motivated by physics, can be applied to provide results about random walks. We only touch upon these connections; see $[\mathbf{7}, \mathbf{2 3}]$ for much more.

Let a nonempty subset $S \subseteq V$ and a function $\pi_{0}: S \rightarrow \mathbb{R}$ be given. We describe three constructions, one in each of the fields mentioned, that extend $\pi_{0}$ to a function $\pi: V \rightarrow \mathbb{R}$ so that the extension is harmonic at the nodes in $V \backslash S$.

ExAmple 1. Let $\pi(v)$ be the expectation of $\pi_{0}(s)$, where $s$ is the (random) node where a random walk on the graph $G$ starting at $v$ first hits $S$.

We can re-state this construction as a discrete version of the Poisson Formula. Let $S \subseteq V(G)$. For every $i \in V(G) \backslash S$ and $j \in S$, let $K(i, j)$ denote the probability that a random walk started at $i$ hits $j$ before any other node in $S$. Then for every function $f$ on $V(G)$ that is harmonic on $V \backslash S$, and every $i \in V \backslash S$

$$
f(i)=\sum_{j \in S} K(i, j) f(j) .
$$

Example 2. Consider the graph $G$ as an electrical network, where each edge represents a unit resistance. Keep each node $s \in S$ at electric potential $\pi_{0}(s)$, and let the electric current flow through $G$. Define $\pi(v)$ as the electric potential of node $v$.

EXAMPLE 3. Consider the edges of the graph $G$ as ideal springs with unit Hooke constant (i.e., it takes $h$ units of force to stretch them to length $h$ ). Nail each node $s \in S$ to the point $\pi_{0}(s)$ on the real line, and let the graph find its equilibrium. The energy is a positive definite quadratic form of the positions of the nodes, and so there is a unique minimizing position, which is the equilibrium. Define $\pi(v)$ as the position of node $v$ on the line.

More generally, fix the positions of the nodes in $S$ (in any dimension), and let the remaining nodes find their equilibrium. Then every coordinate function is harmonic at every node of $V \backslash S$.

A consequence of the uniqueness property is that the harmonic functions constructed (for the case $|S|=2$ ) in examples 1, 2 and 3 are the same. As an application of this idea, we show the following interesting connections (see Nash-Williams [22], Chandra at al. [6]). Let $G$ be a graph with $n$ nodes and $m$ edges. Considering $G$ as an electrical network, let $R_{s t}$ denote the effective resistance between nodes $s$ and $t$. Considering the graph $G$ as a spring structure in equilibrium, with two nodes $s$ and $t$ nailed down at 1 and 0 , let $F_{a b}$ denote the force pulling the nails. Doing a random walk on $G$, let $\kappa(a, b)$ denote the commute time between nodes $a$ and $b$ (i.e., the expected time it takes to start at $a$, walk until you first hit $b$, and then walk until you first hit $a$ again). 
TheOREM 3.3. $R_{a b}=\frac{1}{F_{a b}}=\frac{\kappa(a, b)}{2 m}$.

Using the "topological formulas" from the theory of electrical networks for the resistance, we get a further well-known characterization of these quantities:

COROLlary 3.4. Let $G^{\prime}$ denote the graph obtained from $G$ by identifying a and $b$, and let $\mathcal{T}(G)$ denote the number of spanning trees of $G$. Then

$$
R_{a b}=\frac{\mathcal{T}(G)}{\mathcal{T}\left(G^{\prime}\right)}
$$

\section{Analytic functions on the grid}

4.1. Definition and variations. Suppose that we have an analytic function $g$ on the complex plane, and we can consider its restriction $f$ to the set of lattice points (Gaussian integers) (say, for the purpose of numerical computation). Suppose that we want to "integrate" this function $f$ along a path, which now is a polygon $v_{0} v_{1} \ldots v_{n}$ where $v_{k+1}-v_{k} \in\{ \pm 1, \pm i\}$. A reasonable guess is to use the formula

$$
\sum_{k=0}^{n-1}\left(v_{k+1}-v_{k}\right) \frac{f\left(v_{k+1}\right)+f\left(v_{k}\right)}{2} .
$$

Unfortunately, this sum will in general depend on the path, not just on its endpoints. Of course, the dependence will be small, since the sum approximates the "true" integral.

Can we modify our strategy by defining $f$ not as the restriction of $g$ to the lattice, but as some other discrete approximation of $g$, for which the discrete integral (9) is independent from the path? To answer this question, we have to understand the structure of such discrete functions. Independence from the path means that the integral is 0 on closed paths, which in turn is equivalent to requiring that the integral is 0 on the simplest closed paths of the form $(z, z+1, z+1+i, z+i, z)$. In this case, the condition is

$$
\begin{aligned}
& \frac{f(z+1)+f(z)}{2}+i \frac{f(z+1+i)+f(z+1)}{2} \\
& +(-1) \frac{f(z+i)+f(z+1+i)}{2}+(-i) \frac{f(z)+f(z+i)}{2}=0 .
\end{aligned}
$$

By simple rearrangement, this condition can be written as

$$
\frac{f(z+i+1)-f(z)}{i+1}=\frac{f(z+1)-f(z+i)}{1-i} .
$$

This latter equation can be thought of as discrete version of the fact that the derivative is unique, or (after rotation), as a discrete version of the Cauchy-Riemann equation.

Let $\Omega$ be a subset of the plain that is the union of lattice squares. A function satisfying (10) for every square in $\Omega$ is called a discrete analytic function on $\Omega$. This notion was introduced by Ferrand [11] and developed by Duffin [8]. There are several variations, some of which are equivalent to this, others are not (see e.g. Isaacs [12]).

The following version is essentially equivalent. The lattice of Gaussian integers can be split into "even" and "odd" lattice points $(a+b i$ with $a+b$ even or odd), and condition (10) only relates the differences of even values to the differences of 
odd values. We can take the even sublattice, rotate by $45^{\circ}$, and rescale it to get the standard lattice. We can think of the odd sublattice as the set of fundamental squares of the even lattice. This way a discrete analytic function can be thought of as a pair of complex-valued functions $f_{1}$ and $f_{2}$ defined on the lattice points and on the lattice squares, respectively. These are related by the following condition:

Discrete Cauchy-Riemann, complex version Let $a b$ be an edge of the lattice graph (so $b=a+1$ or $b=a+i)$, and let $p$ and $q$ be the square to bordering ab from the left and right, respectively. Then

$$
f_{1}(b)-f_{1}(a)=i\left(f_{2}(p)-f_{2}(q)\right) .
$$

We call such a pair $\left(f_{1}, f_{2}\right)$ a complex discrete analytic pair.

This form suggests a further simplification: since this equation relates the real part of $f_{1}$ to the imaginary part of $f_{2}$, and vice versa, we can separate these. So to understand discrete analytic functions, it suffices to consider pairs of real valued functions $g_{1}$ and $g_{2}$, one defined on the standard lattice, one on the lattice squares, related by the following condition:

Discrete Cauchy-Riemann, real version Let $a b$ be an edge of the lattice graph ( so $b=a+1$ or $b=a+i)$, and let $p$ and $q$ be the square to bordering ab from the left and right, respectively. Then

$$
g_{1}(b)-g_{1}(a)=g_{2}(p)-g_{2}(q) .
$$

To do computations, it is convenient to label each square by its lower left corner. This way a discrete analytic function can be thought of as two functions $f_{1}$ and $f_{2}$ defined on the lattice points, related by the equations

$$
\begin{gathered}
f_{1}(z+1)-f_{1}(z)=-i\left(f_{2}(z)-f_{2}(z-i)\right), \\
f_{1}(z+i)-f_{1}(z)=i\left(f_{2}(z)-f_{2}(z-1)\right) .
\end{gathered}
$$

In the real version, we get the equations

$$
\begin{aligned}
& g_{1}(x+1, y)-g_{1}(x, y)=g_{2}(x, y)-g_{2}(x, y-1) \\
& g_{1}(x, y+1)-g_{1}(x, y)=g_{2}(x-1, y)-g_{2}(x, y) .
\end{aligned}
$$

Both functions $g$ and $g_{2}$ are harmonic on the infinite graph formed by lattice points, with edges connecting each lattice point to its four neighbors. Indeed,

$$
\begin{aligned}
& {\left[g_{1}(x+1, y)-g_{1}(x, y)\right]+\left[g_{1}(x-1, y)-g_{1}(x, y)\right]} \\
& \quad+\left[g_{1}(x, y+1)-g_{1}(x, y)\right]+\left[g_{1}(x, y-1)-g_{1}(x, y)\right] \\
& =[h(x, y)-h(x, y-1)]+[h(x-1, y-1)-h(x-1, y)] \\
& +[h(x-1, y)-h(x, y)]+[h(x, y-1)-h(x-1, y-1)] \\
& =0 .
\end{aligned}
$$

Conversely, if we are given a harmonic function $g_{1}$, then we can define a function $g_{2}$ on the squares such that $\left(g_{1}, g_{2}\right)$ satisfy (12). We define $g_{2}$ on one square arbitrarily, and then use (12) to extend the definition to all squares. The assumption that $g_{1}$ is harmonic guarantees that we don't run into contradiction by going around a lattice point; since the plane is simply connected, we don't run into contradiction at all.

So we see that a discrete analytic function can be identified with a single complex valued harmonic function on the even sublattice, which in turn can be thought of a 
pair of real valued harmonic functions on the same lattice. To each (real or complex) harmonic function we can compute a conjugate using (11) or (12). It turns out that both ways of looking at these functions are advantageous in some arguments.

4.2. Integration and differentiation. We defined discrete analytic functions so that integration should be well defined: Given a discrete analytic function $f$ and two integer points $a, b$, we can define the integral from $a$ to $b$ by selecting a lattice path $a=z_{0}, z_{1}, \ldots, z_{n}$, and defining

$$
\int_{a}^{b} f d z=\sum_{k=0}^{n-1} \frac{f\left(z_{k+1}\right)+f\left(z_{k}\right)}{2}\left(z_{k+1}-z_{k}\right) .
$$

The main point in our definition of discrete analytic functions was that this is independent of the choice of the path. It is not obvious, but not hard to see, that the integral function

$$
F(u)=\int_{a}^{u} f d z
$$

is a discrete analytic function.

A warning sign that not everything works out smoothly is the following. Suppose that we have two discrete analytic functions $f$ and $g$ defined on $\Omega$. It is natural to try to define the integral

$$
\int_{a}^{b} f d g=\sum_{k=0}^{n-1} \frac{f\left(z_{k+1}\right)+f\left(z_{k}\right)}{2}\left(g\left(z_{k+1}\right)-g\left(z_{k}\right)\right) .
$$

It turns out that this integral is again independent of the path, but it is not an analytic function of the upper bound in general.

There are several ways one could try to define the derivative. The function defined by

$$
\left(\nabla_{a} f\right)(z)=\frac{f(z+a)-f(z)}{a},
$$

is discrete analytic for any Gaussian integer $a(a=i+1$ seems the most natural choice in view of (10)). Unfortunately, neither one if these is the converse of integration. If $F(u)=\int_{a}^{u} f d z$, then for $a \in\{ \pm 1, \pm i\}$,

$$
\left(\nabla_{a} F\right)(z)=\frac{f(z+a)+f(z)}{2} .
$$

There is in fact no unique converse, since adding $c \cdot(-1)^{x+y}$ to the function value at $x+i y$ does not change the integral along any path. This also implies that the converse of integration cannot be recovered "locally". But if we fix the value arbitrarily at (say) 0 , then (13), applied with $a=1$ and $a=i$, can be used to recover the values of $f$ one-by one. This also can be expressed by integration (see $[8])$.

\subsection{Constructions.}

ExAmPle 4 (Extension). To see that there is a large variety of discrete analytic functions, we mention the following fact: if we assign a complex number to every integer point on the real and imaginary axes, there is a unique discrete analytic function with these values. Indeed, we can reconstruct the values of the function at the other integer points $z$ one by one by induction on $|z|^{2}$, using (10). 
EXAMPLE 5 (Discrete polynomials). The restriction of a linear or quadratic polynomial to the lattice points gives a discrete analytic function, but this is not so for polynomials of higher degree. But there are sequences of discrete analytic functions that can be thought of as analogues of powers of $z$.

One of these is best described in terms of an analytic pair. For $n \geq 1$, consider the functions

$$
g_{1}^{\{n\}}(x, y)=n ! \sum_{j=0}^{\lfloor n / 2\rfloor}(-1)^{j}\left(\begin{array}{c}
x-j \\
n-2 j
\end{array}\right)\left(\begin{array}{c}
y+j \\
2 j
\end{array}\right)
$$

and

$$
g_{2}^{\{n\}}(x, y)=n ! \sum_{j=0}^{\lfloor(n-1) / 2\rfloor}(-1)^{j}\left(\begin{array}{c}
x-j \\
n-2 j-1
\end{array}\right)\left(\begin{array}{c}
y+j+1 \\
2 j+1
\end{array}\right)
$$

Then $g_{1}^{\{n\}}$ and $g_{2}^{\{n\}}$ satisfy the conditions (12). (To explain these formulas, note that if we replace $\left(\begin{array}{l}u \\ k\end{array}\right)$ by $u^{k} / k$ !, then we get the real and imaginary parts of $(x+i y)^{n}$.) Taking linear combinations, we get "polynomials". These functions are polynomials in $x$ and $y$, or (after a change of coordinates) in the complex numbers $z$ and $\bar{z}$, but not necessarily polynomials in $z$.

Integration offers another way to define "pseudo-powers" of $z$ :

$$
z^{(0)}=1, z^{(n)}=n \int_{0}^{z} w^{(n-1)} d w .
$$

These functions are not the same as the analytic functions defined by the pairs $\left(g_{1}^{\{n\}}, g_{2}^{\{n\}}\right)$ defined above, but they give rise to the same linear space of discrete polynomials. These functions approximate the true powers of $z$ quite well: Duffin proves that $z^{(n)}-z^{n}$ is a polynomial in $Z$ and $\bar{z}$ of degree at most $n-2$. Hence

$$
z^{(n)}=z^{n}\left(1+O\left(|z|^{-2}\right)\right)
$$

ExAMPLE 6 (Discrete exponentials). Once we have analogues of powers of $z$, we can obtain further discrete analytic functions by series expansion. As an example, we can define the exponential function by the formula

$$
e(z)=\sum_{n=0}^{\infty} \frac{1}{n !} z^{(n)} .
$$

More generally, one can introduce a continuous variable $z$, and define (at least for $|t|<2)$

$$
\left.e(z, t)=\sum_{n=0}^{\infty} \frac{1}{n !} z^{(n)}\right) t^{n}
$$

For this function, Ferrand proved the explicit formula

$$
e(z, t)=\left(\frac{2+t}{2-t}\right)^{x}\left(\frac{2+i t}{2-i t}\right)^{y}
$$

This function is discrete analytic for every fixed $t \neq \pm 2, \pm 2 i$. 
4.4. Approximation. Let go back to the remark we used to motivate discrete analytic functions: that we want to use discrete analytic function to approximate a "true" analytic function by a function on a discrete set of points, in a more sophisticated way than restricting it.

One way to construct such an approximation is to first approximate $f(z)$ by a polynomial $p(z)$ (which could be a partial sum of the Taylor expansion), and then replace $z^{n}$ by $z^{(n)}$ in the polynomial. It follows from (14) that by this, we introduce a relative error of $1+O\left(|z|^{n-2}\right)$. If we do this not on the lattice $L$ of Gaussian integers, but on the lattice $\delta L$ with $\delta \rightarrow 0$, then we get an approximation with relative error $1+O\left(\delta^{2}\right)$. See Duffin and Peterson [10] for details.

While the space of polynomials is well-defined, which polynomials we want to call "powers of $z$ " is a matter of taste, and expansion in terms of other sequences of polynomials may have better properties. For example, Zeilberger [26] constructs another sequence $\left(p_{n}(z)\right)$ for which the series $\sum_{n} a_{n} p_{n}(z)$ converges absolutely to a discrete analytic function in the quadrant $x, y \geq 0$ whenever $\left|a_{n}\right|^{1 / n} \rightarrow 0$.

Many results from complex analysis can be extended (mutatis mutandis) to discrete analytic functions. Besides Cauchy's integral formulas and the Maximum Principle, these include the Phragmén-Lindelöf Theorem, the Paley-WienerSchwartz theorem, and more. See also $[\mathbf{2 7}, \mathbf{2 8}]$ for details.

\section{Holomorphic forms on maps}

While no function can be harmonic at all nodes of a finite graph, the notion of holomorphic forms can be extended to any finite graph embedded in an orientable surface.

5.1. Preliminaries about maps. Let $S$ be a 2-dimensional orientable manifold. By a map on $S$ we mean graph $G=(V, E)$ embedded in $S$ so that

(i) each face is an open topological disc, whose closure is compact,

(ii) every compact subset of $S$ intersects only a finite number of edges.

We in fact will need mainly two cases: either $S$ is the plane or $S$ is compact. In the first case, $G$ is necessarily infinite; in the second, $G$ is finite.

We can describe the map by a triple $G=(V, E, \mathcal{F})$, where $V$ is the set of nodes, $E$ is the set of edges, and $\mathcal{F}$ is the set of faces of $G$. We set $n=|V|, m=|E|$, and $f=|\mathcal{F}|$.

We call an edge $e$ one-sided, if it is incident with one and the same face on both sides, and two-sided otherwise.

For every map $G$, we can construct its universal cover map $\hat{G}=(\hat{V}, \hat{E}, \hat{\mathcal{F}})$ in the usual way. This is an infinite graph embedded in the plane, invariant under the action of an appropriate discrete group of isometries of the euclidean plane (in the case of the torus) or of the hyperbolic plane (in the case of higher genus).

Fixing any reference orientation of $G$, we can define for each edge a right shore $r_{e} \in \mathcal{F}$, and a left shore $l_{e} \in \mathcal{F}$. Recall that an edge $e=i j$ has a head $h_{e}=j$ and a tail $t_{e}=i$.

The embedding of $G$ defines a dual map $G^{*}=\left(V^{*}, E^{*}, \mathcal{F}^{*}\right)$. Geometrically, we create a new node inside each face of $G$, to get $V^{*}$. For each edge $e \in E$, we connect the two faces bordering this edge by an edge $e^{*}$ that crosses $e$ exactly once. (If the same face is incident with $e$ from both sides, then $e^{*}$ is a loop.) It is not hard to arrange these curves so that these new edges give a map $G^{*}$. Combinatorially, we 
can think of $G^{*}$ as the map where "node" and "face" are interchanged, "tail" is replaced "right shore", and "head" is replaced by "left shore". So $\left|E^{*}\right|=|E|$, and there is an obvious bijection $e \leftrightarrow e^{*}$. Note that "right shore" is replaced by "head" and "left shore" is replaced by "tail". So $\left(G^{*}\right)^{*}$ is not $G$, but $G$ with all edges reversed.

Sometimes it is useful to consider another map $G_{\diamond}$ associated with a map $G$, called the diamond map. This is defined on the node set $V\left(G_{\diamond}\right)=V \cup V^{*}$, where the edges are those pairs $x y$ where $x \in V, y \in V *$, and $x$ is incident with the face $F$ corresponding to $y$. (If $F$ has $t$ corners at $x$, we connect $y$ to $x$ by $t$ edges, one through each of these corners.) Clearly $G^{*}$ is a bipartite map where each face has 4 edges.

For every face $F \in \mathcal{F}$, we denote by $\partial F \in \mathbb{R}^{E}$ the boundary of $F$ :

$$
(\partial F)_{e}= \begin{cases}1 & \text { if } r_{e}=F \\ -1 & \text { if } l_{e}=F \\ 0 & \text { otherwise }\end{cases}
$$

Then $d_{F}=|\partial F|^{2}$ is the length of the cycle bounding $F$.

Let $e$ and $f$ be two consecutive edges along the boundary of a face $F$, meeting at a node $v$. We call the quadruple $(F, v, e, f)$ a corner (at node $v$ on face $F$ ). If both edges are directed in or directed out of $b$, we call the corner sharp; else, we call it blunt.

5.2. Circulations, homology and discrete Hodge decomposition. If $G$ is a map on a surface $S$, then the space of circulations on $G$ has an important additional structure: for each face $F$, the vector $\partial F$ is circulation. Circulations that are linear combinations of these special circulations $\partial F$ are called 0 -homologous. Two circulations $\phi$ and $\phi^{\prime}$ are homologous if $\phi-\phi^{\prime}$ is 0 -homologous.

Let $\phi$ be a circulation on $G$. We say that $\phi$ is rotation-free, if for every face $F \in \mathcal{F}$, we have

$$
(\partial F)^{\top} \phi=\sum_{e: r_{e}=F} \phi(e)-\sum_{e: l_{e}=F} \phi(e)=0 .
$$

This is equivalent to saying that $\phi$ is a circulation on the dual map $G^{*}$.

The following linear spaces correspond to the Hodge decomposition. Let $\mathcal{A} \subseteq$ $\overrightarrow{\mathbb{R}}^{E}$ be the subspace generated by the vectors $\delta v(v \in V)$ and $\mathcal{B} \subseteq \mathbb{R}^{E}$, the subspace generated by the vectors $\partial F(F \in \mathcal{F})$. Vectors in $\mathcal{A}$ are sometimes called tensions or potentials. Vectors in $\mathcal{B}$ are 0 -homologous circulations. It is easy to see that $\mathcal{A}$ and $\mathcal{B}$ are orthogonal to each other. The orthogonal complement $\mathcal{A}^{\perp}$ is the space of all circulations, while $\mathcal{B}^{\perp}$ consists of rotation-free vectors on the edges. The intersection $\mathcal{C}=\mathcal{A}^{\perp} \cap \mathcal{B}^{\perp}$ is the space of rotation-free circulations.

Proposition 5.1. For every map $G$ on a surface $S$ with genus $G$, the space of all 1-chains has a decomposition

$$
\overleftarrow{\mathbb{R}^{E}}=\mathcal{A} \oplus \mathcal{B} \oplus \mathcal{C}
$$

into three mutually orthogonal subspaces, where $\mathcal{A}$ is the space of 0-homologous circulations, $\mathcal{B}$ is the space of all potentials, and $\mathcal{C}$ is the space of all rotation-free circulations.

If the map $G$ is not obvious from the context, we denote these spaces by $\mathcal{A}(G), \mathcal{B}(G)$ and $\mathcal{C}(G)$. 
From this proposition we conclude the following.

COROLlaRY 5.2. Every circulation is homologous to a unique rotation-free circulation.

It also follows that $\mathcal{C}$ is isomorphic to the first homology group of $S$ (over the reals), and hence we get the following:

COROLlary 5.3. The dimension of the space $\mathcal{C}$ of rotation-free circulations is $2 g$.

Indeed, we have

$$
\operatorname{dim}(\mathcal{A})=f-1 \quad \text { and } \quad \operatorname{dim}(\mathcal{B})=n-1
$$

by elementary graph theory, and hence

$$
\operatorname{dim}(\mathcal{B})=m-\operatorname{dim}(\mathcal{A})-\operatorname{dim}(\mathcal{B})=m-f-n+2=2 g
$$

by Euler's Formula.

Figure 1 shows the (rather boring) situation on the toroidal grid: for every choice of $a$ and $b$ we get a rotation-free circulation, and by Corollarv 5.3, these are all.

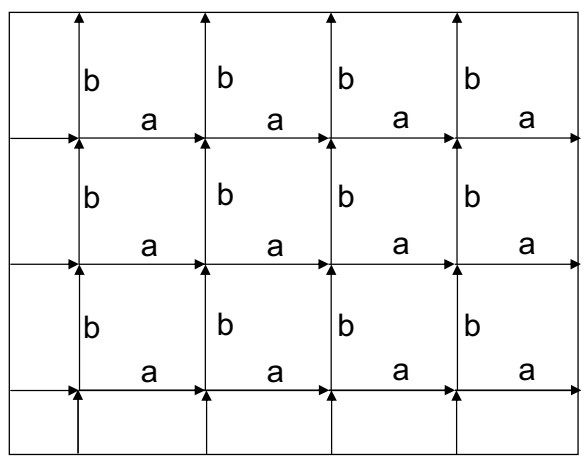

FIGURE 1. Rotation-free circulation on the toroidal grid.

5.3. Discrete analytic functions on a map. To explain the connection between rotation-free circulations and discrete analytic functions, let $\phi$ be a rotationfree circulation on a map $G$ in the plane. Using that $\phi$ is rotation-free, we can construct a function $\pi: \hat{V} \rightarrow \mathbb{R}$ such that

$$
\phi(e)=\pi\left(t_{e}\right)-\pi\left(h_{e}\right)
$$

for every edge $e$. Similarly, the fact that $\phi$ is a circulation implies that there exists a function $\sigma: \hat{\mathcal{F}} \rightarrow \mathbb{R}$ such that

$$
\phi(e)=\sigma\left(r_{e}\right)-\sigma\left(l_{e}\right)
$$

for every edge $e$.

It is easy to see that $\pi$ is harmonic at all nodes of $\hat{G}$ and $\sigma$ is harmonic at all nodes of the dual map. Furthermore, $\pi$ and $\sigma$ are related by the following condition:

$$
\sigma\left(l_{e}\right)-\sigma\left(r_{e}\right)=\pi\left(h_{e}\right)-\pi\left(t_{e}\right) .
$$


for every edge $e$ (since both sides are just $\phi(e))$. We can think of $\pi$ and $\sigma$ as the real and imaginary parts of a (discrete) analytic function. The relation (17) is then a discrete analogue of the Cauchy-Riemann equations.

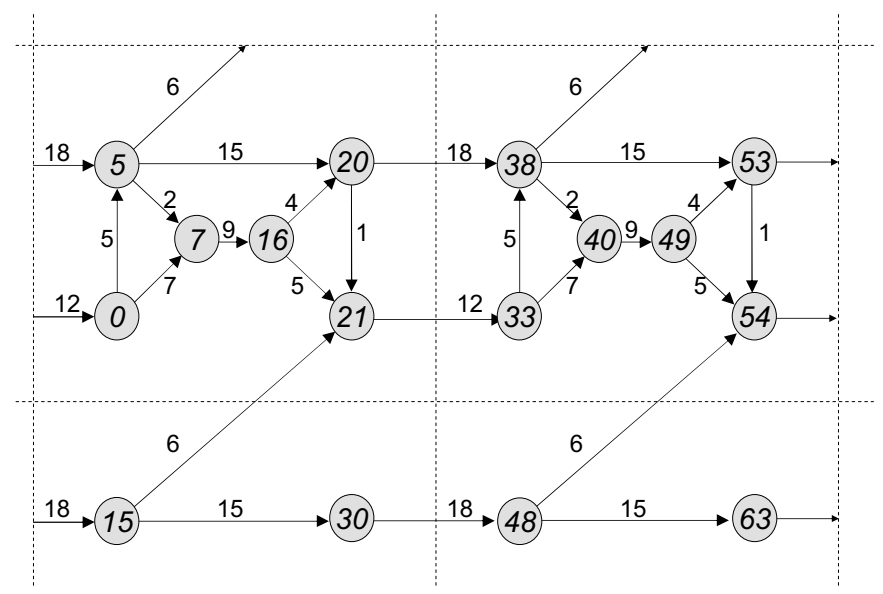

Figure 2. A rotation-free circulation on the torus, and a corresponding harmonic function on the universal cover.

Figures 2 and 3 show a rotation-free circulation on a graph embedded in the torus. The first figure shows how to obtain it from a harmonic function on the nodes of the universal cover map, the second, how to obtain it from a harmonic function on the faces.

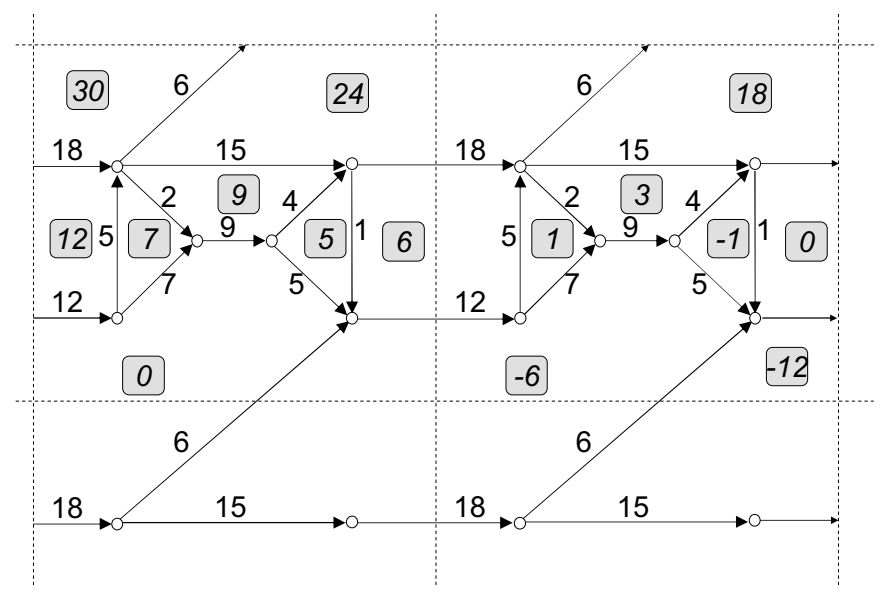

FigURE 3. A harmonic function on the faces of the universal cover associated with the same rotation-free circulation.

As we mentioned in the introduction, discrete analytic functions and holomorphic forms on general maps were introduced by Mercat [18]. His definition is more general than the one above on two counts. First, he allows weighted edges; we'll come back to this extension a bit later. Second, he allows complex values. Let's have a closer look on this. 
Let $G=(V, E, \mathcal{F})$ be a discrete map in the plane, and let $G^{*}=\left(V^{*}, E *, \mathcal{F}^{*}\right)$ be its dual map. Let $f: V \cup V^{*} \rightarrow \mathbf{C}$. We say that $f$ is analytic, if

$$
f\left(l_{e}\right)-f\left(r_{e}\right)=i\left(f\left(h_{e}\right)-f\left(t_{e}\right)\right) .
$$

for every edge $e$. Relation (18) implies a number of further properties; for example, the $f$ to $V$ is harmonic. Indeed, let $y_{1}, \ldots, y_{d}$ be neighbors of $x$ and let $\left(x y_{k}\right) *=$ $p_{k} p_{k+1}$ (where $p_{d+1}=p_{1}$. Then

$$
\sum_{i=1}^{d}\left(f\left(y_{k}\right)-f(x)\right)=\sum_{k=1}^{d} i\left(f\left(p_{k+1}\right)-f\left(p_{k}\right)\right)=0 .
$$

It follows that if $f$ is analytic, then the function $\phi: E \rightarrow \mathbf{C}$ defined by

$$
\phi(e)=f\left(h_{e}\right)-f\left(t_{e}\right),
$$

is a complex valued rotation-free circulation on $G$, which we call a holomorphic form on $G$.

Conversely, for any complex-valued function $\phi: E \rightarrow \mathbf{C}$, we define $\phi^{*}: E^{*} \rightarrow$ C by

$$
\phi^{*}\left(e^{*}\right)=i \cdot \phi(e)
$$

Then $\phi$ is rotation-free if and only if $\phi^{*}$ is a circulation. So if both $\phi$ and $\phi^{*}$ are circulations, then they are both rotation-free. Similarly as in the real case, we can represent both $\phi$ and $\phi^{*}$ as differentials of functions on the nodes and faces, respectively. It is convenient to think of the two primitive functions as a single function $f$ defined on $V \cup V^{*}$. So we have

$$
\phi(e)=f\left(h_{e}\right)-f\left(t_{e}\right), \quad \text { and } \quad \phi^{*}(e)=f\left(l_{e}\right)-f\left(r_{e}\right)
$$

for every edge $e$, and hence $f$ is analytic on the whole map.

However, just like we saw it in the case of discrete analytic functions on a grid, the complex version is not substantially more general than the real. Indeed, a complex-valued function on the edges is a rotation-free circulation if and only if its real and imaginary parts are; and (19) only relates the real part of $\phi$ to the imaginary part of $\phi^{*}$ and vice versa. So a holomorphic form is just a pair of two rotation-free circulations, with no relation between them. In some cases (like in the topological considerations in section 6 ) the real format is more convenient, in others (like defining integration in section 8), the complex format is better.

Let $f$ be a (complex-valued) discrete analytic function on a map in the plane; this corresponds to four real harmonic functions: $\pi_{1}=\Re(f)$ on $G, \pi_{2}=\Im(f)$ on $G, \sigma_{2}=\Re(f)$ on $G^{*}$ and $\sigma_{1}=\Im(f)$ on $G^{*}$. Here $\pi_{1}$ and $\sigma_{1}$ are related by the Cauchy-Riemann equations, and so are $\pi_{2}$ and $\sigma_{2}$; but there is no relation between the pair $\left(\pi_{1}, \sigma_{1}\right)$ and the pair $\left(\pi_{2}, \sigma_{2}\right)$.

This way of looking at analytic functions explains the following construction, which does not seem to correspond to any classical notion. Let us multiply the second pair by -1 ; we get another 4-tuple of harmonic functions satisfying the Cauchy-Riemann equations, which correspond to a complex-valued discrete analytic function $f^{\dagger}$. In other words, we define the conjugate of $f$ by

$$
f^{\dagger}(i)= \begin{cases}\overline{f(i)}, & \text { if } i \in V(G), \\ -\overline{f(i)}, & \text { if } i \in V\left(G^{*}\right) .\end{cases}
$$


This construction was introduced in the special case of grids by Duffin, and it plays an important role in defining derivatives.

5.4. The weighted case. We can consider the following more general setup (Mercat [18]). Suppose that every edge $e$ in the graph as well as in the dual graph has a positive weight $\ell_{e}$ associated with it, which we call its length. This assignment is symmetric, so that $\ell_{i j}=\ell_{j i}$. We can think of the length of the dual edge $e^{*}$ as the "width" of the edge $e$.

For $\phi: E \rightarrow \mathbf{C}$, we define the flow condition as

$$
\sum_{e} \delta v(e) \ell_{e^{*}} \phi(e)=0
$$

and the rotation-free condition for a face $F$ as

$$
\sum_{e} \partial F(e) \ell_{e} \phi(e)=0
$$

(In terms of hydrodynamics, we think of $\phi(e)$ as the speed of flow along the edge e.) This means that

$$
\phi^{*}\left(e^{*}\right)=\phi(e)
$$

defines a rotation-free circulation on the dual graph.

Similarly as before, we can consider real or complex valued circulations, and one complex rotation-free circulation will be equivalent to a pair of real ones.

Consider a complex valued rotation-free circulation $\phi$ on a map in the plane. Then there is a function $f$ on $V \cup V^{*}$ so that for every edge $e$

$$
\phi(e)=\frac{f\left(h_{e}\right)-f\left(t_{e}\right.}{\ell_{e}}=i \frac{f\left(l_{e}\right)-f\left(r_{e}\right)}{\ell_{e^{*}}} .
$$

Such a function $f$ is called a primitive function of $\phi$.

In most of this paper we'll not consider the weighted case, because it would not amount to much more than inserting ' $\ell_{e}$ ' or ' $\ell_{e^{*}}$ ' at appropriate places in the equations. In section 8 , however, choosing the right weighting will be an important issue.

5.5. Constructing holomorphic forms. We give a more explicit construction of rotation-free circulations in the compact case, using electrical currents. For $e=a b \in E$, consider the harmonic function $\pi_{e}$ with poles $a$ and $b$ (as defined in section 3.1). The function $\delta \pi_{e}$ is certainly rotation-free, but it is not a circulation: $a$ is a source and $b$ is a sink (all the other nodes satisfy the flow condition). We could try to repair this by sending a "backflow" along the edge $a b$; in other words, we consider $\pi_{e}-\chi_{e}$. This is now a circulation, but it is not rotation-free around the faces $r_{e}$ and $l_{e}$.

The trick is to also consider the dual map $G^{*}$, the dual edge $(a b)^{*}=p q$, and the harmonic function $\pi_{e^{*}}$. We carry out the same construction as above, to get $\delta^{*} \pi_{e^{*}}$. Then we can combine these to repair the flow condition without creating rotation: We define

$$
\eta_{e}=\delta \pi_{e}-\delta^{*} \pi_{e^{*}}+\chi_{e} .
$$

The considerations above show that $\eta_{e}$ is a rotation-free circulation. In addition, it has the following description:

Lemma 5.4. The circulation $\eta_{e}$ is the orthogonal projection of $\chi_{e}$ to the space $\mathcal{C}$ of rotation-free circulations. 
Proof. It suffices to show that

$$
\chi_{e}-\eta_{e}=\delta \pi_{e}-\delta^{*} \pi_{e^{*}}
$$

is orthogonal to every $\phi \in \mathcal{C}$. But $\delta \pi_{e} \in \mathcal{A}$ by $\left(2\right.$, and similarly, $\delta^{*} \pi_{e^{*}} \in \mathcal{B}$. So both are orthogonal to $\mathcal{C}$.

This lemma has some simple but interesting consequences. Since the vectors $\chi_{e}$ span $\mathbb{R}^{E}$, their projections $\eta_{e}$ generate the space of rotation-free circulations. Since $\eta_{e}$ is a projection of $\chi_{e}$, we have

$$
\eta_{e}(e)=\eta_{e} \cdot \chi_{e}=\left|\eta_{e}\right|^{2} \geq 0 .
$$

Let $R_{e}$ denote the effective resistance between the endpoints of an edge $e$, and let $R_{e^{*}}$ denote the effective resistance of the dual map between the endpoints of the dual edge $e^{*}$. Then we get by Theorem 3.3 that

$$
\eta_{e}(e)=1-R_{e}-R_{e^{*}} .
$$

If we work with a map on the sphere, we must get 0 by Theorem 5.3 . This fact has the following consequence (which is well known, and can also be derived e.g. from Corollary 3.4): for every planar map, $R_{e}+R_{e^{*}}=1$. For any other underlying surface, we get $R_{e}+R_{e^{*}} \leq 1$. It will follow from theorem 6.6 below that strict inequality holds here, as soon as the map satisfies some mild conditions.

\section{Topological properties}

6.1. Combinatorial singularities. We need a simple combinatorial lemma about maps on compact surfaces. For every face $F$, let $a_{F}$ denote the number of sharp corners of $F$. For every node $v$, let $b_{v}$ denote the number of blunt corners at $v$. So $a_{F}$ is the number of times the orientation changes if we move along the boundary of $F$, while $b_{v}$ is the number of times the orientation changes in the cyclic order of edges as they emanate from $v$.

Lemma 6.1. Let $G=(V, E, \mathcal{F})$ be any digraph embedded on a surface $S$ with Euler characteristic $\chi$. Then

$$
\sum_{F \in \mathcal{F}}\left(a_{F}-2\right)+\sum_{v \in V}\left(b_{v}-2\right)=4 g-4 .
$$

PROOF. Counting sharp corners, we get

$$
\sum_{F} a_{F}=\sum_{v}\left(d_{v}-b_{v}\right)
$$

and so by Euler's formula,

$$
\sum_{F} a_{F}+\sum_{v} b_{v}=\sum_{v} d_{v}=2 m=2 n+2 f+4 g-4 .
$$

Rearranging, we get the equality in the lemma.

Suppose that the orientation of the map is such that there are no sources and sinks (so each node has at least one edge going out and at least one edge going in), and no face boundary is a directed cycle. Then $b_{v} \geq 2$ for each node and $a_{F} \geq 2$ for each face, and so every term in (24) is nonnegative. Lemma 6.1 says that all but at most $2 g-2$ nodes will have $b_{v}=2$, which means that both the incoming edges and the outgoing edges are consecutive in the cyclic ordering around the 
node. Similarly, all but at most $2 g-2$ faces will have $a_{F}=2$, which means that the face boundary consists of two directed paths.

6.2. Zero sets. Some useful nondegeneracy properties of rotation-free circulations were proved in $[\mathbf{2}, \mathbf{3}]$. In this section we present these in a more general form.

An analytic function cannot vanish on a open set, unless it is identically 0 . What is the corresponding statement for finite graphs? For which subgraphs of a map can we find a discrete holomorphic form that vanishes on all edges of the subgraph? In other words, what do we know about the support subgraph $H_{\phi}$ of a rotation-free circulation?

Let $H$ be a subgraph of a finite connected graph $G$. Consider the connected components of $G \backslash V(H)$. A bridge of $H$ is defined as a subgraph of $G$ that consists of one of these components, together with all edges connecting it to $H$, and their endpoints in $H$. We also consider edges not in $H$ but connecting two nodes in $H$ as trivial bridges of $H$. Let $\mathcal{B}(H)$ denote the set of bridges of $H$. For every bridge $B \in \mathcal{B}(H)$, we call its nodes in $H$ its terminals. The other nodes of the bridge are called internal.

If we look at a small neighborhood of a terminal $v$ of $B$, then the edges of $H$ incident with this node divide this neighborhood into "corners". The bridge $B$ may have edges entering $v$ through different corners. The number of corners $B$ uses is the multiplicity of the terminal. We denote the sum of multiplicities of all terminals of $B$ by $\tau(B)$.

THEOREM 6.2. Let $H$ be a subgraph of a map $G$ on an orientable surface $S$ with genus $g$.

(a) If $H$ is the support of a rotation-free circulation $\phi$, then

$$
\sum_{\substack{B \in \mathcal{B}(H) \\ \tau(B) \geq 2}}(\tau(B)-2) \leq 4 g-2 .
$$

(b) If

$$
\sum_{B \in \mathcal{B}(H)}(\tau(B)-1) \leq 2 g-1,
$$

then there is a rotation-free circulation with support contained in $H$.

Let us make some remarks in connection with this theorem.

1. Part (b) of the theorem implies that a rotation-free circulation can vanish on a rather large part of the graph, which could contain even the majority of the nodes. It is not the size of a set $S$ that matters, but rather how well connected $S$ is to the non-trivial parts of the graph.

2. It would be nice to replace $\tau(B)-2$ by $\tau(B)-1$ in (a); but no such result can be stated, since we cannot control the number of trivial bridges. As an example, consider the rotation-free circulation in Figure 1 on a toroidal grid with $a=1, b=0$.

3. If $H$ satisfies (a) but not (b), then whether or not there exists a nonzero rotation-free circulation supported on $H$ may depend on finer properties. An interesting case is a node of degree 6 in a map on the double torus $(g=2)$. One can construct examples where no rotation-free circulation can vanish on all 6 edges, and other examples where it can. 
Let us formulate some corollaries of this theorem. The following theorem was proved in $[\mathbf{2}, \mathbf{3}]$. We say that a connected subgraph $H$ is $k$-separable in $G$, if $G$ can be written as the union of two graphs $G_{1}$ and $G_{2}$ so that $\left|V\left(G_{1}\right) \cap V\left(G_{2}\right)\right| \leq k$, $V(H) \cap V\left(G_{2}\right)=\emptyset$, and $G_{2}$ contains a non-0-homologous cycle.

Corollary 6.3. Let $G$ be a map on an orientable surface $S$ of genus $g>0$, and let $H$ be a connected subgraph of $G$. If $H$ is not $(4 g-1)$-separable in $G$, then every rotation-free circulation vanishing on all edges of $\bar{H}$ is identically 0 .

Corollary 6.4. Assume that for every set $X$ of $4 g-1$ nodes, all but one of the components of $G-X$ are plane and have fewer than $k^{\prime}$ nodes. Let $H$ be a connected subgraph with $k$ nodes. Then every rotation-free circulation vanishing on all edges incident with $H$ is identically 0.

A map is called $k$-representative, if every non-contractible Jordan curve on the surface intersects the map in at least $k$ points.

Corollary 6.5. Let $G$ be a $(4 g-1)$-representative map on an orientable surface $S$ of genus $g>0$. Then every rotation-free circulation vanishing on all edges of a non-0-homologous cycle, and on all edges incident with it, is identically 0 .

6.3. Identically zero-sets. Most of the time, the motivation for the study of discrete analytic functions is to transfer the powerful methods from complex analysis to the study of graphs. In this section we look at questions that are natural for graphs. It would be interesting to find analogues or applications in the continuous setting.

Recall that for every oriented edge $e$, we introduced the rotation-free circulation $\eta_{e}$. We want to give a sufficient condition for this projection to be non-zero. The fact that $\eta_{e}$ is the orthogonal projection of $\chi_{e}$ to $\mathcal{C}$ implies that the following three assertions are equivalent: $\eta_{e} \neq 0 ; \eta_{e}(e) \neq 0$; there exists a rotation-free circulation $\phi$ with $\phi(e) \neq 0$.

THEOREM 6.6. Let $G$ be a 3-connected simple map an orientable surface with genus $g>0$. Then $\eta_{e} \neq 0$ for every edge $e$.

The toroidal graphs in Figure 4 (where the surrounding area can be any graph embedded in the torus) show that the assumption of 3-connectivity and the exclusion of loops and parallel edges cannot be dropped ${ }^{1}$

COROLlary 6.7. If $G$ is a 3-connected simple graph on a surface with positive genus, then there exists a nowhere-0 rotation-free circulation.

Another corollary gives an explicit lower bound on the entries of $\eta_{a b}$.

COROLlary 6.8. If $G$ is a 3-connected simple graph on a surface with positive genus, then for every edge $e, \eta_{e}(e) \geq n^{2-n} f^{2-f}$.

Indeed, combining Theorem 6.6 with (22), we see that $\eta_{e}(e)>0$ if $g>0$. But $\eta_{e}(e)=1-R_{e}-R_{e^{*}}^{*}$ is a rational number, and from Theorem 3.4 it follows that its denominator is not larger than $n^{n-2} f^{f-2}$.

COROLlaRY 6.9. If $G$ is a 3-connected simple graph on a surface with positive genus, then for every edge $e, R_{e}+R_{e^{*}}^{*}<1$.

${ }^{1}$ This condition was erroneously omitted in $[\mathbf{3}]$. 

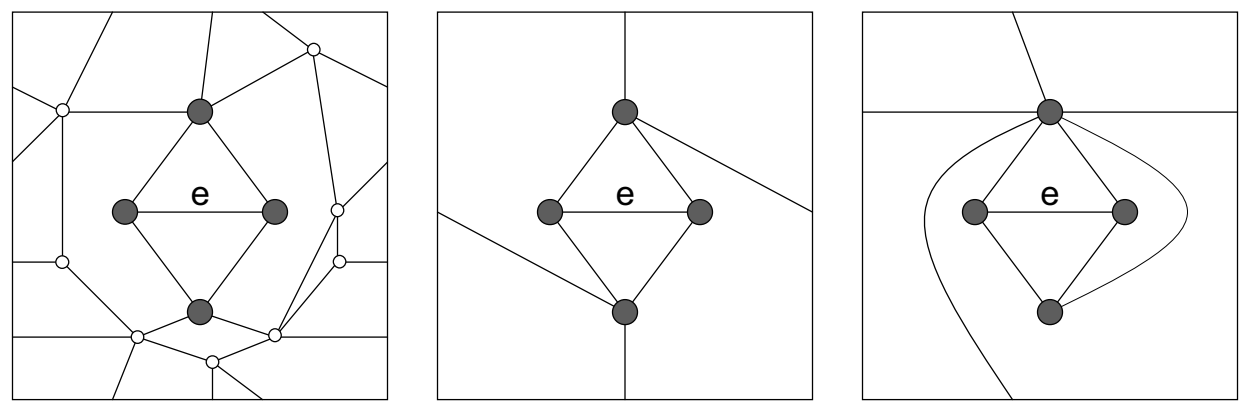

Figure 4. Every rotation-free circulation is 0 on the edge $e$.

6.4. Generic independence. The question whether every rotation-free circulation vanishes on a given edge is a special case of the following: given edges $e_{1}, \ldots, e_{k}$, when can we independently prescribe the values of a rotation-free circulation on them? Since the dimension of $\operatorname{dim}(\mathcal{C})=2 g$, we must have $k \leq 2 g$. There are other obvious conditions, like the set should not contain the boundary of a face or the coboundary of a node. But a complete answer appears to be difficult.

We get, however, a question that can be answered, if we look at the "generic" case: we consider the weighted version, and assume that there is no numerical coincidence, by taking (say) algebraically independent weights. Using methods from matroid theory, a complete characterization of such edge-sets can be given [17]. For example, the following theorem provides an NP-coNP characterization and (through matroid theory) a polynomial algorithm in the case when $k=2 g$. We denote by $c(G)$ the number of connected components of the graph $G$.

THEOREM 6.10. Let $W \subseteq E$ be any set of $2 g$ edges of a map on an orientable surface with genus $g$, with algebraically independent weights. Then the following are equivalent:

(a) Every set of prescribed values on $W$ can be extended to a rotation-free circulation in a unique way.

(b) $E(G)-W$ can be partitioned into two sets $T$ and $T^{*}$ so that $T$ forms a spanning tree in $G$ and $T^{*}$ forms a spanning tree in $G^{*}$.

(c) For every set $W \subseteq S \subseteq E(G)$ of edges

$$
c(G \backslash S)+c\left(G^{*} \backslash S\right) \leq|S|+2-2 g .
$$

As another special case, one gets that for a given edge $e$, there is a rotation-free circulation that is non-zero on $e$ for some weighting (equivalently, for almost all weightings) of the edges if and only if the map contains a non-zero-homologous cycle through $e$.

\section{Geometric connections}

7.1. Straight line embeddings. We can view a (complex-valued) analytic function $f$ on a map $G$ as a mapping of the nodes into the complex plane. We can extend this to the whole graph by mapping each edge $u v$ on the segment connecting $f(u)$ and $f(v)$. It turns out that under rather general conditions, this mapping is an embedding. To formulate the condition, note that on the nodes of $G$ we can define a distance $d_{G}(u, v)$ as the minimum length of path in $G$ connecting $u$ and $v$. 
TheOREM 7.1. Let $G$ be a simple 3-connected map in the plane and let $f$ be an analytic function on $G$. Suppose that there exist a constant $c$ such that

$$
\frac{1}{c} \leq \frac{|f(u)-f(v)|}{d_{G}(u, v)} \leq c .
$$

for every pair of distinct nodes $u, v \in V \cup V^{*}$. Then $f$ defines an embedding. Furthermore, this embedding has the additional property that every face is a convex polygon, and every node is in the center of gravity of its neighbors.

One case when the conditions in the theorem are automatically fulfilled is when the map $G$ is the universal cover map of a toroidal map $H$ and $f$ is the primitive function of a holomorphic form on $H$. Then the embedding defined by $f$ can be "rolled up" to the torus again. So we obtain the following corollary:

COROLlary 7.2. Every holomorphic form on a simple 3-connected toroidal map defines an embedding of it in the torus such that all edges are geodesic arcs.

7.2. Square tilings. A beautiful connection between square tilings and rotationfree flows was described in the classic paper of Brooks, Smith, Stone and Tutte [5]. They considered tilings of squares by smaller squares, and used the connection with flows to construct a tiling of a square with squares whose edge-lengths are all different. For our purposes, periodic tilings of the whole plane are more relevant.

Consider tiling of the plane with squares, whose sides are parallel to the coordinate axes. Assume that the tiling is discrete, i.e., every bounded region contains only a finite number of squares. We associate a map in the plane with this tiling as follows. Represent any maximal horizontal segment composed of edges of the squares by a single node (say, positioned at the midpoint of the segment). Each square "connects" two horizontal segments, and we can represent it by an edge connecting the two corresponding nodes, directed top-down. We get an (infinite) directed graph $G$ (Figure 5).

It is not hard to see that $G$ is planar. If we assign the edge length of each square to the corresponding edge, we get a circulation: If a node $v$ represents a segment $I$, then the total flow into $v$ is the sum of edge length of squares attached to $I$ from the top, while the total flow out of $v$ is the sum of edge length of squares attached to $I$ from the bottom. Both of these sums are equal to the length of $I$ (let's ignore the possibility that $I$ is infinite for the moment). Furthermore, since the edge-length of a square is also the difference between the $y$-coordinates of its upper and lower edges, this flow is rotation-free.

Now suppose that the tiling is double periodic with period vectors $a, b \in \mathbb{R}^{2}$ (i.e., we consider a square tiling of the torus). Then so will be the graph $G$, and so factoring out the period, we get a map on the torus. Since the tiling is discrete, we get a finite graph. This also fixes the problem with the infinite segment $I$ : it will become a closed curve on the torus, and so we can argue with its length on the torus, which is finite now. The flow we constructed will also be periodic, so we get a rotation-free circulation on the torus.

We can repeat the same construction using the vertical edges of the squares. It is not hard to see this gives the dual graph, with the dual rotation-free circulation on it.

A little attention must be paid to points where four squares meet. Suppose that $A, B, C, D$ share a corner $p$, where $A$ is the upper left, and $B, C, D$ follow clockwise. In this case, we must consider the lower edges of $A$ and $B$ to belong to a single 


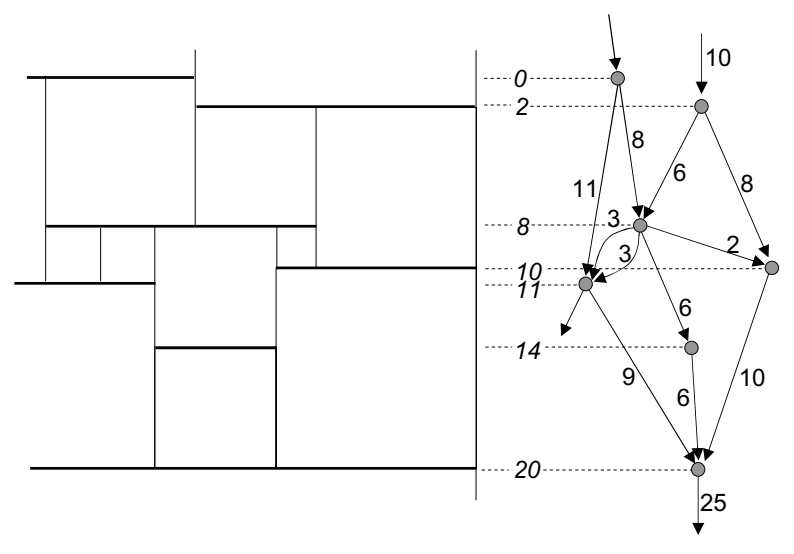

Figure 5. The Brooks-Smith-Stone-Tutte construction

horizontal segment, but interrupt the vertical segment at $p$, or vice versa. In other words, we can consider the lower edges of $A$ and $C$ "infinitesimally overlapping".

This construction can be reversed. Take a toroidal map $G^{*}$ and any rotationfree circulation on it. Then this circulation can be obtained from a doubly periodic tiling of the plane by squares, where the edge-length of a square is the flow through the corresponding edge. (We suppress details.)

If an edge has 0 flow, then the corresponding square will degenerate to s single point. Luckily, we know (Corollary 6.7) that for a simple 3-connected toroidal map, there is always a nowhere-zero rotation-free circulation, so these graphs can be represented by a square tiling with no degenerate squares.

7.3. Rubber bands. Another important geometric method to represent planar graph was described by Tutte [25]. Tutte used it to obtain drawings of planar graphs, but we apply the method to toroidal graphs.

Let $G$ be a toroidal map. We consider the torus as $\mathbb{R}^{2} / \mathbf{Z}^{2}$, endowed with the metric coming from the euclidean metric on $\mathbb{R}^{2}$. Let us replace each edge by a rubber band, and let the system find its equilibrium. Topology prevents the map from collapsing to a single point. In mathematical terms, we are minimizing

$$
\sum_{i j \in E(G)} \ell(i j)^{2}
$$

where the length $\ell(i j)$ of the edge $i j$ is measured in the given metric, and we are minimizing over all continuous mappings of the graph into the torus homomorphic to the original embedding.

It is not hard to see that the minimum is attained, and the minimizing mapping is unique up to isometries of the torus. We call it the rubber band mapping. Clearly, the edges are mapped onto geodesic curves. A nontrivial fact is that if $G$ is a simple 3 -connected toroidal map, then the rubber band mapping is an embedding. This follows from Theorem 7.1.

We can lift this optimizing embedding to the universal cover space, to get a planar map which is doubly periodic, and the edges are straight line segments. Moreover, every node is at the center of gravity of its neighbors. This follows 
simply from the minimality of (26). This means that both coordinate functions are harmonic and periodic, and so their coboundaries are rotation-free circulations on the original graph. Since the dimension of the space $\mathcal{C}$ of rotation-free circulations on a toroidal map is 2 , this construction gives us the whole space $\mathcal{C}$.

This last remark also implies that if $G$ is a simple 3-connected toroidal map, then selecting any basis $\phi_{1}, \phi_{2}$ in $\mathcal{C}$, the primitive functions of $\phi_{1}$ and $\phi_{2}$ give a doubly periodic straight-line embedding of the universal cover map in the plane.

7.4. Circle representations. Our third geometric construction that we want to relate to discrete holomorphic forms are circle representations. A celebrated theorem of Koebe [15] states that the nodes of every planar graph can be represented by openly disjoint circular discs in the plane, so that edges correspond to tangency of the circles. Andre'ev [1] improved this by showing that there is a simultaneous representation of the graph and its dual. Thurston [24] extended this to the toroidal graphs, and this is the version we need.

It is again best to go to the universal cover map $\hat{G}$. Then the result says that for every 3 -connected toroidal graph $G$ we can construct two (infinite, but discrete) families $\mathcal{F}$ and $\mathcal{F}^{*}$ of circles in the plane so that they are double periodic modulo a lattice $L=\mathbf{Z} a+\mathbf{Z} b, \mathcal{F}(\bmod L)$ corresponds to the nodes of $G, \mathcal{F}^{*}(\bmod L)$ corresponds to the faces of $G$, and for ever edge $e$, there are two circles $C, C^{\prime}$ representing $h_{e}$ and $t_{e}$, and two circles $D$ and $D^{\prime}$ representing $r_{e}$ and $l_{e}$ so that $C, C^{\prime}$ are tangent at a point $p, D, D^{\prime}$ are tangent at the same point $p$, and $C, D$ are orthogonal.

If we consider the centers the circles in $\mathcal{F}$ as nodes, and connect two centers by a straight line segment if the circles touch each other, then we get a straight line embedding of the universal cover map in the plane (appropriately periodic modulo $L)$. Let $f(i)$ denote the point representing node $i$ of the universal cover map. or of its dual.

To get a holomorphic form out of this representation, consider the plane as the complex plane, and define $\phi(i j)=\rho(j)-\rho(i)$ for every edge of $\hat{G}$ or $\hat{G}^{*}$. Clearly $\phi$ is invariant under $L$, so it can be considered as a function on $E(G)$. By the orthogonality property of the circle representation, $\phi(e) / \phi\left(e^{*}\right)$ is a positive multiple of $i$. In other words,

$$
\frac{\phi(e)}{|\phi(e)|}=i \frac{\phi\left(e^{*}\right)}{\left|\phi\left(e^{*}\right)\right|}
$$

It follows that if we consider the map $G$ with weights

$$
\ell_{e}=|\phi(e)|, \quad \ell_{e^{*}}=\left|\phi\left(e^{*}\right)\right|,
$$

then $\phi$ is a discrete holomorphic form on this weighted map.

It would be nice to be able to turn this construction around, and construct a circle representation using discrete holomorphic forms.

\section{Operations}

8.1. Integration. Let $f$ and $g$ be two functions on the nodes of a discrete weighted map in the plane. Integration is easiest to define along a path $P=$ 
$\left(v_{0}, v_{1}, \ldots, v_{k}\right)$ in the diamond graph $G_{\diamond}$ (this has the advantage that it is symmetric with respect to $G$ and $\left.G^{*}\right)$. We define

$$
\int_{P} f d g=\sum_{i=0}^{k-1} \frac{1}{2}\left(f\left(v_{i+1}\right)+f\left(v_{i}\right)\right)\left(g\left(v_{i+1}\right)-g\left(v_{i}\right)\right) .
$$

The nice fact about this integral is that for analytic functions, it is independent of the path $P$, depends on the endpoints only. More precisely, let $P$ and $P^{\prime}$ be two paths on $G_{\diamond}$ with the same beginning node and endnode. Then

$$
\int_{P} f d g=\int_{P^{\prime}} f d g .
$$

This is equivalent to saying that

$$
\int_{P} f d g=0
$$

if $P$ is a closed path. It suffices to verify this for the boundary of a face of $G_{\diamond}$, which only takes a straightforward computation. It follows that we can write

$$
\int_{u}^{v} f d g
$$

as long as the homotopy type of the path from $u$ to $v$ is determined (or understood).

Similarly, it is also easy to check the rule of integration by parts: If $P$ is a path connecting $u, v \in V \cup V^{*}$, then

$$
\int_{P} f d g=f(v) g(v)-f(u) g(u)-\int_{P} g d f .
$$

Let $P$ be a closed path in $G_{\diamond}$ that bounds a disk $D$. Let $f$ be an analytic function and $g$ an arbitrary function. Define $\hat{g}(e)=g\left(h_{e}\right)-g\left(t_{e}\right)-i\left(g\left(l_{e}\right)-g\left(r_{e}\right)\right)$ (the "analycity defect" of $g$ on edge $e$. Then it is not hard to verify the following generalization of (28):

$$
\int_{P} f d g=\sum_{e \subset D}\left(f\left(h_{e}\right)-f\left(t_{e}\right)\right) \hat{g}(e) .
$$

This can be viewed as a discrete version of the Residue Theorem. For further versions, see [18].

Kenyon's ideas in $[\mathbf{1 3}]$ give a nice geometric interpretation of (28). Let $G$ be a map in the plane and let $g$ be an analytic function on $G$. Let us assume that $g$ satisfies the conditions of Theorem 7.1, so that it gives a straight-line embedding of $G$ in the plane with convex faces, and similarly, a straight-line embedding of $G^{*}$ with convex faces. Let $P_{u}$ denote the convex polygon representing the face of $G$ (or $G^{*}$ ) corresponding to $u \in V^{*}($ or $u \in V)$ ). Shrink each $F_{u}$ from the point $g(u)$ by a factor of 2 . Then we get a system of convex polygons where for every edge $u v \in G_{\diamond}$, the two polygons $P_{u}$ and $P_{v}$ share a vertex at the point $(g(u)+g(v)) / 2$ (Figure 6(a)). There are two kinds of polygons (corresponding to the nodes in $V$ and $V^{*}$, respectively. It can be shown that the interiors of the polygons $P_{u}$ will be disjoint (the point $g(u)$ is not necessarily in the interior of $P_{u}$ ). The white areas between the polygons correspond to the edges of $G$. They are rectangles, and the sides of the rectangle corresponding to edge $e$ are $g\left(h_{e}\right)-g\left(t_{e}\right)$ and $g\left(l_{e}\right)-g\left(r_{e}\right)$. 

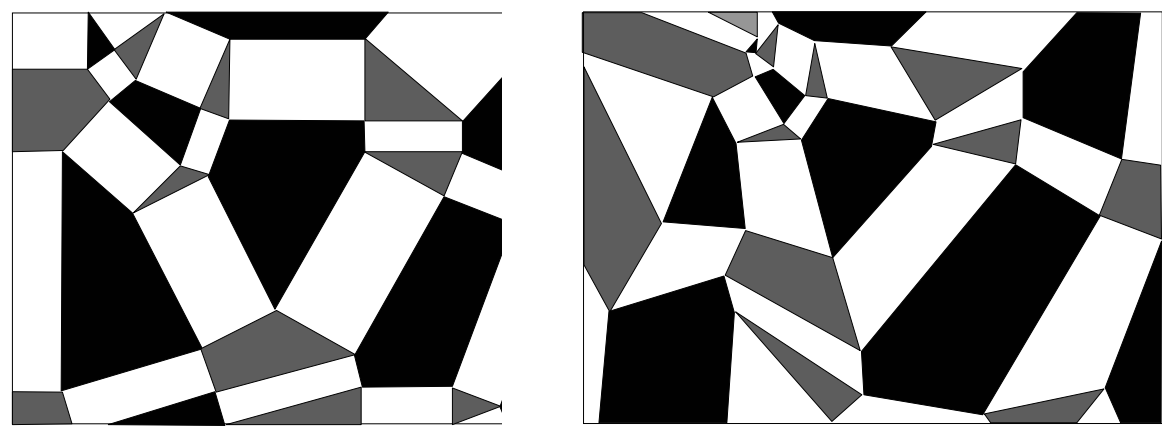

FiguRE 6. Representation of an analytic function by touching polygons, and a deformation given by another analytic function.

Now take the other analytic function $f$, and construct the polygons $f(u) P_{u}$ (multiplication by the complex number $f(u)$ corresponds to blowing up and rotating). The resulting polygons will not meet at the appropriate vertices any more, but we can try to translate them so that they do. Now equation (28) tells us that we can do that (Figure 6(b)). Conversely, every "deformation" of the picture such that the polygons $P_{u}$ remain similar to themselves defines an analytic function on $G$.

8.2. Critical analytic functions. These have been the good news. Now the bad part: for a fixed starting node $u$, the function

$$
F(v)=\int_{u}^{v} f d g
$$

is uniquely determined, but it is not analytic in general. In fact, a simple computation shows that for any edge $e$,

$$
\begin{aligned}
\hat{F}(e) & =\frac{F\left(h_{e}\right)-F\left(t_{e}\right)}{\ell_{e}}-i \frac{F\left(l_{e}\right)-F\left(r_{e}\right)}{\ell_{e^{*}}} \\
& =i \frac{f\left(h_{e}\right)-f\left(t_{e}\right)}{\ell_{e}}\left[g\left(t_{e}\right)+g\left(h_{e}\right)-g\left(r_{e}\right)-g\left(l_{e}\right)\right] .
\end{aligned}
$$

So we want an analytic function $g$ such that the factor in brackets in (31) is 0 for every edge:

$$
g\left(t_{e}\right)+g\left(h_{e}\right)=g\left(r_{e}\right)+g\left(l_{e}\right)
$$

Let us call such an analytic function critical. What we found above is that $\int_{u}^{v} f d g$ is an analytic function of $v$ for every analytic function $f$ if and only if $g$ is critical.

This notion was introduced in a somewhat different setting by Duffin [9] under the name of rhombic lattice. Mercat [18] defined critical maps: these are maps which admit a critical analytic function.

Geometrically, this condition means the following. Consider the function $g$ as a mapping of $G \cup G^{*}$ into the complex plane $\mathbf{C}$. This defines embeddings of $G, G^{*}$ and $G \diamond$ in the plane with following (equivalent) properties:

(a) The faces of $G^{\diamond}$ are rhomboids.

(b) Every edge of $G_{\diamond}$ has the same length.

(c) Every face of $G$ is inscribed in a unit circle. 
(d) Every face of $G^{*}$ is inscribed in a unit circle.

Criticality can be expressed in terms of holomorphic forms as well. Let $\phi$ be a (complex valued) holomorphic form on a weighted map $G$. We say that $\phi$ is critical if the following condition holds: Let $e=x y$ and $f=y z$ be two edges of $G$ bounding a corner at $y$, with (say) directed so that the corner is on their left, then

$$
\ell_{e} \phi(e)+\ell_{f} \phi(f)=\ell_{e^{*}} \phi\left(e^{*}\right)-\ell_{f^{*}} \phi\left(f^{*}\right) .
$$

Note that both $f$ and $f^{*}$ are directed into $h_{f}$, which explains the negative sign on the right hand side. To digest this condition, consider a plane piece of the map and a primitive function $g$ of $\psi$. Then (33) means that

$$
g\left(y^{\prime}\right)-g(y)=g(q)-g\left(q^{\prime}\right)
$$

which we can rewrite in the following form:

$$
g(x)+g(y)-g(p)-g(q)=g(x)+g\left(y^{\prime}\right)-g(p)-g\left(q^{\prime}\right) .
$$

This means that $g\left(h_{e}\right)+g\left(t_{e}\right)-g\left(l_{e}\right)-g\left(r_{e}\right)$ is the same for every edge $e$, and since we are free to add a constant to the value of $g$ at every node in $V^{*}$ (say), we can choose the primitive function $g$ so that $g$ is critical.

Whether or not a weighted map in the plane has a critical holomorphic form depends on the weighting. Which maps can be weighted this way? A recent paper of Kenyon and Schlenker [14] answers this question. Consider any face $F_{0}$ of the diamond graph $G_{\diamond}$, and a face $F_{1}$ incident with it. This is a quadrilateral, so there is a well-defined face $F_{2}$ so that $F_{0}$ and $F_{2}$ are attached to $F_{1}$ along opposite edges. Repeating this, we get a sequence of faces $\left(F_{0}, F_{1}, F_{2} \ldots\right)$. Using the face attached to $F_{0}$ on the opposite side to $F_{1}$, we can extend this to a two-way infinite sequence $\left(\ldots, F_{-1}, F_{0}, F_{1}, \ldots\right)$. We call such a sequence a track.

TheOREM 8.1. A planar map has a rhomboidal embedding in the plane if and only if every track consists of different faces and any two tracks have at most one face in common.

8.3. Polynomials, exponentials, derivation and approximation. Once we can integrate, we can define polynomials. More exactly, let $G$ be a map in the plane, and let us select any node to be called 0 . Let $Z$ denote a critical analytic function on $G$ such that $Z(0)=0$. Then we have

$$
\int_{0}^{x} 1 d Z=Z(x) .
$$

Now we can define higher powers of $Z$ by repeated integration:

$$
Z^{: n:}(x)=n \int_{0}^{x} Z^{: n-1:} d Z .
$$

We can define a discrete polynomial of degree $n$ as any linear combination of $1, Z, Z^{: 2:}, \ldots, Z^{: n}$. The powers of $Z$ of course depend on the choice of the origin, and the formulas describing how it is transformed by shifting the origin are more complicated than in the classical case. However, the space of polynomials of degree see is invariant under shifting the origin $[\mathbf{1 9}]$ ).

Further, we can define the exponential function $\exp (x)$ as a discrete analytic function $\operatorname{Exp}(x)$ on $V \cap V^{*}$ satisfying

$$
d \operatorname{Exp}(x)=\operatorname{Exp}(x) d Z .
$$


More generally, it is worth while to define a 2-variable function $\operatorname{Exp}(x, \lambda)$ as the solution of the difference equation

$$
d \operatorname{Exp}(\lambda, x)=\lambda \operatorname{Exp}(\lambda, x) d Z .
$$

It can be shown that there is a unique such function, and there are various more explicit formulas, including

$$
\operatorname{Exp}(\lambda, x)=\sum_{n=0}^{\infty} \frac{Z^{: n}}{n !},
$$

(at least as long as the series on the right hand side is absolute convergent).

We can also define derivation, using the notion of the conjugate function defined in (20). Given a (complex) analytic function on (say) a map in the plane, we define

$$
f^{\prime}(j)=\left(\int_{0}^{j} f^{\dagger} d g\right)^{\dagger} .
$$

Then it is not hard to see that $f^{\prime}$ is analytic, and

$$
\int_{0}^{v} f^{\prime} d g=f-f(0)
$$

Mercat $[\mathbf{1 9}, \mathbf{2 0}]$ uses these tools to show that exponentials form a basis for all discrete analytic functions, and to generalize results of Duffin, Zeilberger and others about approximability of analytic functions by discrete analytic functions.

\section{An application in computer science: Global information from local observation}

Suppose that we live in a (finite) map on a compact orientable surface with genus $g$ (we assume the embedding is reasonably dense). On the graph, a random process is going on, with local transitions. Can we determine the genus $g$, by observing the process in a small neighborhood of our location?

Discrete analytic functions motivate a reasonably natural and simple process, called the noisy circulator, which allows such a conclusion. This was constructed by Benjamini and the author [2]. Informally, this can be described as follows. Each edge carries a real weight. With some frequency, a node wakes up, and balances the weights on the edges incident with it, so that locally the flow condition is restored. With the same frequency, a face wakes up, and balances the weights on the edges incident with it, so that the rotation around the face is cancelled. Finally, with a much lower frequency, an edge wakes up, and increases or decreases its weight by 1 .

To be precise, we consider a finite graph $G$, embedded on an orientable surface $S$, so that each face is a disk bounded by a simple cycle. We fix a reference orientation of $G$, and a number $0<p<1$. We start with the vector $x=0 \in \mathbb{R}^{E}$. At each step, the following two operations are carried out on the current vector $x \in \mathbb{R}^{E}$ :

(a) [Node balancing.] We choose a random node $v$. Let $a=(\delta v)^{\top} x$ be the "imbalance" at node $v$ (the value by which the flow condition at $v$ is violated). We modify $f$ by subtracting $\left(a / d_{v}\right) \delta v$ from $x$.

(b) [Face balancing.] We choose a random face $F$. Let $r=(\partial F)^{\top} x$ be the rotation around $F$. We modify $f$ by subtracting $\left(r / d_{F}\right) \partial F$ from $x$. 
In addition, with some given probability $p>0$, we do the following:

(c) [Excitation.] We choose a random edge $e$ and a random number $X \in$ $\{-1,1\}$, and add $X$ to $x_{e}$.

Rotation-free circulations are invariant under node and face balancing. Furthermore, under repeated application of (a) and (b), any vector converges to a rotation-free circulation.

Next we describe how we observe the process. Let $U$ be a connected subgraph of $G$, which is not $(4 g-1)$-separable in $G$. Our observation window is the set $E_{0}$ of edges incident with $U$ (including the edges of $U$ ). Let $x(t) \in \mathbb{R}^{E}$ be the vector of edge-weights after $t$ steps, and let $y(t)$ be the restriction of $x(t)$ to the edges in $E_{0}$. So we can observe the sequence random vectors $y(0), y(1), \ldots$

The main result of $[\mathbf{2}]$ about the noisy circulator is the following (we don't state the result in its strongest form).

TheOREM 9.1. Assume that we know in advance an upper bound $N$ on $n+m+f$. If $p=O\left(N^{-8}\right)$, then observing the Noisy Circulator for $O\left(N^{8} / p\right)$ steps, we can determine the genus $g$ with high probability.

The idea behind the recovery of the genus $g$ is that if the excitation frequency $p$ is sufficiently small, then most of the time $x(t)$ will be essentially a rotation-free circulation. The random excitations guarantee that over sufficient time we get $2 g$ linearly independent rotation-free circulations. Corollary 6.3 implies that even in our small window, we see $2 g$ linearly independent weight assignments $y(t)$.

\section{Acknowledgement}

I am indebted to Oded Schramm, Lex Schrijver and Miki Simonovits for many valuable discussions while preparing this paper.

\section{Appendix}

Proof of Theorem 6.2. (a) Consider an edge $e$ with $\phi(e)=0$. There are various ways $e$ can be eliminated. If $e$ is two-sided, then we can delete $e$ and get a map on the same surface with a rotation-free flow on it. If $e$ is not a loop, then we can contract $e$ and get a map on the same surface with a rotation-free flow on it. If $e$ is a one-sided loop, we can change $\phi(e)$ to any non-zero value and still have a rotation-free circulation.

Of course, we don't want to eliminate all edges with $\phi=0$, since then we don't get anything. We eliminate two-sided edges that constitute trivial bridges (this does not change the assertion in (a)). We contract edges that connect two different internal nodes in the same bridge, so that we may assume that every bridge has exactly one internal node. If there are two edges with $\phi=0$ that together bound a disc (which necessarily connect the internal node of a bridge to a terminal), we delete one of them. We delete any two-sided loop with $\phi=0$. Finally, if we have a one-sided loop with $\phi=0$ attached at a node of $H$, then we send non-zero flow through it arbitrarily.

Let $v_{B}$ denote the internal node of bridge $B$. The node $v_{B}$ has $\tau(B)$ edges connecting it to $H$ (there may be some one-sided loops left that are attached to $\left.v_{B}\right)$. For every face $F$, let $\beta(F)$ denote the number of times we switch between $H$ and the rest when walking along the boundary. 
We need some additional terminology. We call a corner unpleasant if it is at a node of $H$, and both bounding edges are outside $H$. Note that these edges necessarily belong to different bridges. Let $u(v)$ and $u(F)$ denote the number of unpleasant corners at node $v$ and face $F$, respectively. Clearly $\sum_{v} u(v)=\sum_{F} u(F)$ is the total number of unpleasant corners.

Re-orient each edge of $H$ in the direction of the flow $\phi$. Re-orient each edge not in $H$ randomly, independently of each other, with probability $1 / 2$ in either direction. We evaluate the expectation of various terms in Lemma 6.1. A corner that has at most one edge of $H$ will be sharp with probability $1 / 2$ and blunt with probability $1 / 2$. Hence for the internal nodes we have

$$
\mathrm{E}\left(b_{v_{B}}-2\right)=\frac{\tau(B)}{2}-2 .
$$

We claim that for each node $v \in V(H)$,

$$
\mathrm{E}\left(b_{v}-2\right) \geq \frac{u(v)}{2} .
$$

Trivially, $v$ is never a source or a sink, and so $b_{v}-2 \geq 0$ for any of the random orientations. So we may assume that $u(v) \geq 1$. $H$ has at least two blunt corners at $v$. If all the bridges attached at $v$ come in through the same corner, then one of these blunt corners is left intact in every orientation, and the $u(v)$ unpleasant corners give rise to $u(v)+2$ corners at $v$ bordered by at least one edge with $\phi=0$. This means that

$$
\mathrm{E}\left(b_{v}-2\right) \geq 1+\frac{u(v)+2}{2}-2=\frac{u(v)}{2} .
$$

If the bridges attached at $v$ come in through at least two corners, then the $u(v)$ unpleasant corners give rise to at least $u(v)+4$ corners at $v$ bordered by at least one edge with $\phi=0$, and so

$$
\mathrm{E}\left(b_{v}-2\right) \geq \frac{u(v)+4}{2}-2=\frac{u(v)}{2} .
$$

Finally, we claim that for any face $F$

$$
\mathrm{E}\left(a_{F}-2\right) \geq \frac{\beta(F)-u(F)}{2} .
$$

The number of internal points and their neighbors on the boundary of $F$ is at least $3 \beta(F)-u(F)$, and so

$$
\mathrm{E}\left(a_{F}-2\right) \geq \frac{3 \beta(F)-u(F)}{2} .
$$

So if $\beta(F) \geq 2$, then we are done. Suppose that $\beta(F)=1$. If the boundary contains edges with $\phi \neq 0$, then it must contain two such edges that are oriented in the opposite direction (since $\phi$ is rotation-free). There is an expected number of at least $3 / 2$ orientation changes on the arc between these two edges that contains an internal node, and at least one on the other. This gives that

$$
\mathrm{E}\left(a_{F}-2\right) \geq 1+\frac{3}{2}-2=\frac{1}{2}=\frac{\beta(F)-u(F)}{2} .
$$

Finally, if $\beta(F)=0$, then all we have to show is that the face is not oriented. If the face has at least one edge with $\phi \neq 0$ then this is obvious, since $\phi$ is rotation-free. If all its edges have $\phi=0$, then these cannot connect $H$ to $v_{B}$, which means that they must be one-sided. But then the face boundary passes through them twice in the opposite direction, so the face is not oriented. 
Now we have, by Lemma 6.1 ,

$$
\begin{aligned}
4 g-4 & =\mathrm{E}\left(\sum_{F}\left(a_{F}-2\right)+\sum_{v}\left(b_{v}-2\right)\right) \\
& =\sum_{F} \mathrm{E}\left(a_{v}-2\right)+\sum_{v} \mathrm{E}\left(b_{v}-2\right) \\
& \geq \sum_{F} \frac{\beta(F)-u(F)}{2}+\sum_{v \in V(H)} \frac{u(v)}{2}+\sum_{B \in \mathcal{B}(H)}\left(\frac{\tau(B)}{2}-2\right) \\
& =\sum_{F} \frac{\beta(F)}{2}+\sum_{B \in \mathcal{B}(H)}\left(\frac{\tau(B)}{2}-2\right) \\
& =\sum_{B \in \mathcal{B}(H)}(\tau(B)-2),
\end{aligned}
$$

as claimed.

(b) Contract the internal nodes of every non-trivial bridge $B$ to a single node $v_{B}$. If we have two edges in $E(G) \backslash E(H)$ that bound a disc, we delete one of them. So we will have $\tau(B)$ edges connecting $v_{B}$ to $H$. Let $G$ denote the resulting map.

Let $S$ be a set of edges that contains all the trivial bridges and also all but one edge between $v_{B}$ and $H$ for every bridge $B$. Then

$$
|S|=\sum_{B}(\tau(B)-1) \leq 2 g-1 .
$$

Since the space $\mathcal{C}\left(G^{\prime}\right)$ of rotation-free circulations has dimension $2 g$, there is a $\phi \in \mathcal{C}\left(G^{\prime}\right)$ that is 0 on all edges of $S$. From the flow condition it follows that $\phi$ is 0 on the remaining edges between the nodes $v_{B}$ and $H$. Now we can construct a $\psi \in \mathcal{C}(G)$ by keeping $\phi$ on $H$ and extending it by 0 values to the rest of $G$.

Proof of Theorem 6.6. Suppose that $\eta_{e}=0$. Then by the definition (21) of $\eta_{e}$, we have

$$
\pi_{e}\left(h_{f}\right)-\pi_{e}\left(t_{f}\right)=\pi_{e^{*}}\left(r_{f}\right)-\pi_{e^{*}}\left(l_{f}\right)
$$

for every edge $f \neq e$, but

$$
\pi_{e}\left(h_{e}\right)-\pi_{e}\left(t_{e}\right)=\pi_{e^{*}}\left(r_{e}\right)-\pi_{e^{*}}\left(l_{e}\right)-1 .
$$

It will be convenient to set $\alpha(f)=\pi_{e}\left(h_{f}\right)-\pi_{e}\left(t_{f}\right)$. We may choose the reference orientation so that $\alpha(f) \geq 0$ for every edge.

Let $U_{c}$ denote the union of faces $F$ with $\pi_{e^{*}}(F)>c$. The boundary of $U_{c}$ is an eulerian subgraph, and so it can be decomposed into edge-disjoint cycles $D_{1}, \ldots, D_{t}$. For every edge $f \in E\left(D_{j}\right), f \neq e$ we have $\alpha(f)>0$ by (37), and all these edges are oriented in the same way around the cycle. So $\pi_{e}$ strictly increases as we traverse the cycle $D_{j}$. This is clearly a contradiction unless $t=1$ and $e$ is an edge of $D_{1}$. Let $D(c)$ denote this unique boundary cycle of $U_{c}$. It also follows that all the values of $\pi_{e}$ on this cycle are different.

Let $G_{0}$ denote the subgraph formed by those edges $f$ for which $\alpha(f)=0$, and $G_{1}$ the subgraph formed by the other edges. Clearly $\pi_{e}$ is constant on every connected component of $G_{0}$. Hence (i) a cycle $D(c)$ meets a component of $G_{0}$ at most once. 
Next we show that (ii) every node is in $G_{1}$. Suppose $u \notin V\left(G_{1}\right)$. Since $D(c) \subseteq G_{1}$ for any $c$, and there are no parallel edges, the subgraph $G_{1}$ must have at least 3 nodes. By 3 -connectivity, there are 3 paths connecting $u$ to three nodes $v_{1}, v_{2}, v_{3} \in V\left(G_{1}\right)$, which are disjoint from each other except for $u$ and from $G_{1}$ except for the $v_{i}$. All edges incident with inner points of these paths have $\alpha(f)=0$, and hence all faces $F$ incident with inner points of these paths have the same $\pi_{e^{*}}(F)=c$. On the other hand, each $v_{i}$ must be incident with an edge with $\alpha(f) \neq 0$, and hence also with a face $F_{i}$ with $\pi_{e^{*}}\left(F_{i}\right) \neq c$. We may assume by symmetry that $\pi_{e^{*}}\left(F_{1}\right)<c$ and $\pi_{e^{*}}\left(F_{2}\right)<c$. But then $D(c)$ passes through $v_{1}$ and $v_{2}$, which belong to the same component of $G_{0}$, a contradiction.

Essentially the same argument shows that (iii) no two edges of $G_{0}$ form a corner.

We apply Lemma 6.1. Clearly $b_{h_{e}}=b_{t_{e}}=0$. We claim that

$$
b_{v} \leq 2
$$

for every other node. Suppose not, then there are four edges $e_{1}, e_{2}, e_{3}, e_{4}$ incident with $v$ in this clockwise order, so that $h_{e_{1}}=h_{e_{3}}=v$ and $t_{e_{2}}=t_{e_{3}}=v$. Choose these edges so that as many of them as possible belong to $G_{1}$. Then (iv) no two consecutive edges of these four can be in $G_{0}$; indeed, by (iii) there would be an edge $f$ of $G_{1}$ between them, and we could replace one of them by $f$.

First, suppose that all four of these edges belong to $G_{1}$. We may assume that there is no edge of $G_{1}$ between $e_{1}$ and $e_{2}$, nor between $e_{3}$ and $e_{4}$. Then all faces $F$ between $e_{1}$ and $e_{2}$ have the same $\pi_{e^{*}}(F)=c$, and all faces $F$ between $e_{3}$ and $e_{4}$ have the same $\pi_{e^{*}}(F)=c^{\prime}$. Let (say) $c<c^{\prime}$, then $D(c)$ passes through $v$ twice, which is impossible.

Second, suppose that $e_{1} \in E\left(G_{0}\right)$. Then $e_{2}, e_{4} \in E\left(G_{1}\right)$ by (iv). The edge $f$ that follows $e_{1}$ in the clockwise order around $v$ must be in $G_{1}$ by (iii). This edge cannot be directed into $v$, since then we could replace $e_{1}$ by it and decrease the number of $G_{0}$-edges among the four. So we can replace $e_{2}$ by $f$. Thus we may assume that $e_{1}$ and $e_{2}$ form a corner, and similarly for $e_{1}$ and $e_{4}$. Let $\pi_{e^{*}}\left(l\left(e_{1}\right)\right)=$ $\pi_{e^{*}}\left(l\left(e_{1}\right)\right)=c$, then $\pi_{e^{*}}\left(r\left(e_{2}\right)\right)>c$ and $\pi_{e^{*}}\left(r l\left(e_{4}\right)\right)<c$. Let $u=t\left(e_{1}\right)$, then (ii) implies that there is a face $F$ with $\pi_{e^{*}}(F) \neq c$ incident with $u$. Let, say, $\pi_{e^{*}}(F)<c$, then $D(c)$ passes through both endpoints of $e_{1}$, which contradicts $(*)$. This completes the proof of (39).

A similar argument shows that

$$
a_{F} \leq 2
$$

for every face. So substituting in Lemma 6.1 yields $-4 \geq 4 g-4$, or $g \leq 0$, a contradiction.

Proof of Theorem 7.1. The assertion that every node is in the center of gravity of its neighbors is just a restatement of the fact that every analytic function is harmonic. This also shows that (assuming that that $f$ gives an embedding) no face can have a concave angle, and so the faces are convex polygons. So the main step is to show that $f$ defines an embedding.

We start with observing that the image of every edge of $G$ is a segment of length at most $c$, and for any two nodes $u$ and $v$ of $G$ (adjacent or not) the distance of $f(u)$ and $f(v)$ is at least $1 / c$.

We start with some lemmas. 
Lemma 9.2. The image of $G$ is not contained in a single line.

Proof. Suppose that a line $\ell$, say the $x$-axis, contains the image of $G$. Let $V^{\prime}=\left\{v \in V(G): f(v) \geq 0\right.$ and $V^{\prime \prime}=\{v \in V(G): f(v)<0\}$. It follows from (25) that $f(V)$ is a discrete set, and the fact that each node is mapped to the center of gravity of its neighbors implies that both these sets must be infinite. It is not hard to see that there must be infinitely many disjoint edges $u_{k} v_{k}(k=1,2, \ldots)$ of $G$ connecting $V^{\prime}$ and $V^{\prime \prime}$. But then $\left|f\left(u_{k}\right)-f\left(v_{k}\right)\right|$ could not remain bounded, which contradicts (25).

LEMMA 9.3. Let $H$ be an open halfplane an $v$ a node of $G$ such that $f(v) \in H$. Then there exists an infinite path $P_{v}=\left(v_{0}=v, v_{1}, \ldots\right.$ starting at $v$ such that $f\left(P_{v}\right) \subset H$ and the distance of $f\left(v_{k}\right)$ from the boundary of $H$ tends to $\infty$.

Proof. Let $G^{\prime}$ be the subgraph spanned by those nodes that can be reached from $v$ on a path $Q$ such that $f(Q)$ lies in $H$. It suffices to show that the distance of points of $f\left(G^{\prime}\right)$ from the boundary of $H$ is unbounded, since then the existence of $P_{v}$ follows by simple compactness.

Suppose that $f\left(G^{\prime}\right)$ lies in a strip $S$ of finite width, and let $U$ denote the connected component of $\mathbf{C} \backslash f\left(G^{\prime}\right)$ containing the halfplane $H \backslash S$. The boundary of $U$ is a polygon, whose vertices are points $f(i), i \in V\left(G^{\prime}\right)$ and intersection points of images of edges. Neither type of vertices can give a concave angle, and so $U$ is convex; since $U$ contains a halfplane, it follows that $U$ is a halfplane. So its boundary is a line $\ell$.

Clearly $G$ has nodes whose image is on $\ell$ (infinitely many such nodes, in fact). Furthermore, if a node $v$ of $G$ is mapped onto $\ell$, then so are all its neighbors (otherwise, $v$ could not be their center of gravity), so it follows that all nodes of $G$ are mapped onto $\ell$. But this contradicts Lemma 9.2.

LEMma 9.4. For every (open or closed) halfplane $H$, the set $S=\{i \in V(G): f(i) \in$ $H\}$ induces a connected subgraph.

Proof. We may assume that $H$ is the halfplane $\{y \geq 0\}$. Let $u$ and $v$ be two nodes in $S$, we want to show that they can be connected by a path whose image stays in the halfplane $H$. Consider any path $P$ in $G$ connecting $u$ and $v$. We may assume that $P$ is not just an edge, and that all the inner nodes of this path are outside $H$. Let $w$ be a node on $P$ which is lowest.

By Lemma 9.3, we can find three infinite paths $P_{u}, P_{v}$ and $P_{w}$, starting at $u$, $v$ and $w$, respectively, such that $f\left(P_{u}\right)$ and $f\left(P_{v}\right)$ lie in the upper halfplane, $f\left(P_{w}\right)$ lies in the lower halfplane, and the distance from the $x$-axis tends to infinity along each of these paths. Clearly $P_{w}$ is node-disjoint from $P_{u}$ and $P_{v}$. If $P_{u}$ and $P_{v}$ intersect, then the conclusion is trivial, so assume that they are node-disjoint.

Consider $P \cup P_{u} \cup P_{v} \cup P_{w}$ in the original planar embedding of $G$. This subgraph splits the plane into three infinite regions; let $\Omega$ be the region bounded by $P_{u} \cup P \cup P_{v}$. It is easy to see that there are infinitely many disjoint paths $Q_{1}, Q_{2}, \ldots$ connecting $P_{u}$ and $P_{v}$ inside $\Omega$. We claim that if $k$ is large enough, the image of $Q_{k}$ must stay in $H$, proving that $u$ and $v$ can be connected by a path in $S$.

Let $D$ denote the diameter of $f(P)$. Let $u_{1}$ be the last node on the path $P_{u}$ such that the distance of $f\left(u_{1}\right)$ from the $x$-axis is at most $D+2 c^{3}$, let $P_{u}^{\prime}$ be the piece of $P_{u}$ between $u$ and $u_{1}$, and let $P_{u}^{\prime \prime}=P_{u} \backslash P_{u}^{\prime}$. We define $P_{v}^{\prime}, P_{w}^{\prime}$ etc. analogously.

Suppose that $f\left(P_{u}^{\prime \prime}\right)$ intersects $f\left(P_{v}^{\prime \prime}\right)$, say edge $i j$ of $f\left(P_{u}^{\prime \prime}\right)$ intersects edge $a b$ of $f\left(P_{v}^{\prime \prime}\right)$. By (25), the length of the image of any edge is at most $c$, so $|f(i)-f(a)| \leq 2 c$, 
and so $d_{G}(i, a) \leq 2 c^{2}$. Thus there exists a path $R$ of length at most $2 c^{2}$ in $G$ connecting $i$ to $a$. Again by (25), the diameter of $f(R)$ is at most $2 c^{3}$. By the definition of $P_{u}^{\prime \prime}$, the distance of $f(i)$ from the $x$-axis is more than $2 c^{3}$, so $f(R)$ cannot cross the $x$-axis. It follows that $u$ and $v$ can be connected by a path whose image stays in the upper halfplane, using paths $P_{u}, R$, and $P_{v}$.

So we may assume that $f\left(P_{u}^{\prime \prime}\right)$ and $f\left(P_{v}^{\prime \prime}\right)$ are disjoint. Let $T$ be the set of all nodes in $G$ at a graph-distance at most $c_{2}$ from $P \cup P_{u}^{\prime} \cup P_{v}^{\prime} \cup P_{w}^{\prime}$. Since $T$ is a finite set, there is a $k$ for which $Q_{k}$ does not intersect $T$. By (25) we get that for every node $s$ of $Q_{k}$ and every node $t$ of $P$,

$$
|f(s)-f(t)| \geq \frac{d_{G}(s, t)}{c} \geq \frac{c_{1}}{c}=c_{2} .
$$

In particular, $f(s)$ cannot be in the convex hull of $f(P)$.

If $f\left(Q_{k}\right)$ does not intersect the lower halfplane, then we are done. Suppose it does, then either it intersects $f\left(P_{w}^{\prime}\right)$ or else it contains a subpath $Q_{k}^{\prime}$ such that $f\left(Q_{k}^{\prime}\right)$ lies in the upper halfplane and intersects both $f\left(P_{u}^{\prime \prime}\right)$ and $f\left(P_{v}^{\prime \prime}\right)$.

Suppose that $f\left(Q_{k}\right)$ intersects $f\left(P_{w}\right)$. Similarly as above, we find a path $R$ of length at most $2 c^{2}$ in $G$ connecting a node $a$ on $P_{w}$ to a node $i$ on $Q_{k}$. This path must intersect the path $P \cup P_{u} \cup P_{v}$ at some node $z$; this means that $d_{G}(z, a) \leq 2 c^{2}$, and so $|f(z)-f(a)| \leq 2 c^{3}$. But $f(z)$ is either in the upper halfplane or at a distance at most $D$ from it, and so $f(a)$ is at a distance at most $2 c^{3}+D$ from the upper halfplane. So $a \in V\left(P_{u}^{\prime}\right)$ and hence $i \in T$, a contradiction, since $Q_{k}$ avoids $T$.

Finally, if there is a path $Q$ such that $f(Q)$ lies in the upper halfplane and intersects both $f\left(P_{u}^{\prime \prime}\right)$ and $f\left(P_{v}^{\prime \prime}\right)$, then similarly as above, we find two paths $R_{u}$ and $R_{v}$ of length at most $2 c^{2}$ connecting $Q$ to $P_{u}^{\prime \prime}$ and $P_{v}^{\prime \prime}$, respectively. Similarly as above, these paths must stay in the upper halfplane, and so again we find that $u$ and $v$ can be connected by a path staying in the upper halfplane through $P_{u}, R_{u}$, $Q, R_{v}$ and $P_{v}$.

Now we turn to the proof that $f$ defines an embedding. Let us triangulate each face of $G$ arbitrarily, to get a new graph $G_{1}$. Let us draw the images of these new edges as straight segments. We claim that even with these new edges, $f$ defines an embedding.

It is enough to show that (a) the images of every triangular face is a triangle (it does not degenerate), (b) two triangular faces of $G_{1}$ sharing an edge $x y$ are mapped onto triangles on different sides of the line $f(x) f(y)$, and (c) the images of triangular faces incident with the same node $x$ cover a neighborhood of $f(x)$ exactly once. We describe the proof of (b); the proof of (a) and (c) is similar.

So suppose that $x y z$ and $x y w$ are two triangular faces of $G_{1}$, and that $f(z)$ and $f(w)$ are on the same side of the line $\ell$ through $f(x)$ and $f(y)$, say on the right side. By Lemma 9.4, there is a path $P$ in $G$ connecting $z$ and $w$ whose image under $f$ stays on the right side of $\ell$. Since $x$ is mapped to the center of gravity of its neighbors, there is a node $x^{\prime}$ adjacent to $x$ in $G$ such that $f\left(x^{\prime}\right)$ lies on the left side of $\ell$, and similarly, $y$ has a neighbor $y^{\prime}$ such that $f\left(y^{\prime}\right)$ lies on the left side of $\ell$. Again by Lemma 9.4, there is a path $Q$ in $G$ connecting $x^{\prime}$ to $y^{\prime}$ such that the image of $Q$ stays on the left side of $\ell$. Extend $Q$ with the edges $x x^{\prime}$ and $y y^{\prime}$ to get a path $Q^{\prime}$.

Now obviously $P$ and $Q^{\prime}$ are node-disjoint paths. But if we consider them in the planar embedding of $G$, it is clear that they must cross each other. This contradiction proves the theorem. 


\section{References}

[1] E. Andre'ev: On convex polyhedra in Lobachevsky spaces, Mat. Sbornik, Nov. Ser. 81 (1970), 445-478.

[2] I. Benjamini and L. Lovász: Global Information from Local Observation, Proc. 43rd Ann. Symp. on Found. of Comp. Sci. (2002), 701-710.

[3] I. Benjamini and L. Lovász: Harmonic and analytic frunctions on graphs, Journal of Geometry 76 (2003), 3-15.

[4] I. Benjamini and O. Schramm: Harmonic functions on planar and almost planar graphs and manifolds, via circle packings, Invent. Math. 126 (1996), 565-587.

[5] R.L. Brooks, C.A.B. Smith, A.H. Stone, W.T. Tutte: The dissection of rectangles into squares, Duke Math. J. 7 (1940), 312-340.

[6] A.K. Chandra, P. Raghavan, W.L. Ruzzo, R. Smolensky and P. Tiwari: The electrical resistance of a graph captures its commute and cover times, Proc. 21st ACM STOC (1989), $574-586$.

[7] P.D. Doyle and J.L. Snell: Random walks and electric networks, Math. Assoc. of Amer., Washington, D.C. 1984.

[8] R.J. Duffin: Basic properties of discrete analytic functions, Duke Math. J. 23 (1956), 335-363.

[9] R.J. Duffin: Potential theory on the rhombic lattice, J. Comb. Theory 5 (1968) 258-272.

[10] R.J. Duffin and E.L. Peterson: The discrete analogue of a class of entire functions, J. Math. Anal. Appl. 21 (1968) 619-642.

[11] J. Ferrand: Fonctions préharmoniques et fonctions préholomorphes, Bull. Sci. Math. 68 (1944), 152-180.

[12] R. Isaacs, Monodiffric functions, Natl. Bureau Standards App. Math. Series 18 (1952), 257266.

[13] R. Kenyon: The Laplacian and Dirac operators on critical planar graphs, Inventiones Math 150 (2002), 409-439.

[14] R. Kenyon and J.-M. Schlenker: Rhombic embeddings of planar graphs with faces of degree 4 (math-ph/0305057).

[15] P. Koebe: Kontaktprobleme der konformen Abbildung, Berichte über die Verhandlungen d. Sächs. Akad. d. Wiss., Math.-Phys. Klasse, 88 (1936) 141-164.

[16] R. Kenyon and J.-M. Schlenker: Rhombic embeddings of planar graphs (2003) (preprint)

[17] L. Lovász and A. Schrijver (unpublished)

[18] C. Mercat: Discrete Riemann surfaces and the Ising model, Comm. Math. Phys. 218 (2001), 177-216.

[19] C. Mercat: Discrete polynomials and discrete holomorphic approximation (mathph/0206041).

[20] C. Mercat: Exponentials form a basis for discrete holomorphic functions (math-ph/0210016).

[21] B. Mohar and C. Thomassen: Graphs on surfaces. Johns Hopkins Studies in the Mathematical Sciences. Johns Hopkins University Press, Baltimore, MD, 2001.

[22] C. St.J. A. Nash-Williams: Random walks and electric currents in networks, Proc. Cambridge Phil. Soc. 55 (1959), 181-194.

[23] P.M. Soardi: Potential Theory on Infinite Networks, Lecture notes in Math. 1590, SpringerVerlag, Berlin-Heidelberg, 1994.

[24] W.P. Thurston: Three-dimensional Geometry and Topology, Princeton Mathematical Series 35, Princeton University Press, Princeton, NJ, 1997.

[25] W.T. Tutte: How to draw a graph, Proc. London Math. Soc. 13 (1963), 743-768.

[26] D. Zeilberger: A new basis for discrete analytic polynomials, J. Austral. Math. Soc. Ser. A 23 (1977), 95-104.

[27] D. Zeilberger: A new approach to the theory of discrete analytic functions, J. Math. Anal. Appl. 57 (1977), 350-367.

[28] D. Zeilberger and H. Dym: Further properties of discrete analytic functions, J. Math. Anal. Appl. 58 (1977), 405-418.

Microsoft Research, One Microsoft Way, Redmond, WA 98052, USA 